\title{
Systematic search for stellar pulsators in the eclipsing binaries observed by Kepler
}

\author{
Patrick Gaulme ${ }^{1,2}$ and Joyce A. Guzik ${ }^{3}$
}

\author{
${ }^{1}$ Max-Planck-Institut für Sonnensystemforschung, Justus-von-Liebig-Weg 3, 37077 Göttingen, Germany \\ e-mail: gaulme@mps .mpg.de \\ 2 Department of Astronomy, New Mexico State University, PO Box 30001, MSC 4500, Las Cruces, NM 88003-8001, USA \\ 3 Los Alamos National Laboratory, XTD-NTA, MS T086, Los Alamos, NM 87545-2345, USA
}

Received 2 May 2019 / Accepted 16 August 2019

\begin{abstract}
Eclipsing binaries (EBs) are unique targets for measuring precise stellar properties and can be used to constrain stellar evolution models. In particular, it is possible to measure masses and radii of both components of a double-lined spectroscopic EB at the percent level. Since the advent of high-precision photometric space missions (MOST, CoRoT, Kepler, BRITE, TESS), the use of stellar pulsation properties to infer stellar interiors and dynamics constitutes a revolution for studies of low-mass stars. The Kepler mission has led to the discovery of thousands of classical pulsators such as $\delta$ Scuti and solar-like oscillators (main sequence and evolved), but also almost $3000 \mathrm{EBs}$ with orbital periods shorter than 1100 days. We report the first systematic search for stellar pulsators in the entire Kepler EB catalog. The focus is mainly aimed at discovering $\delta$ Scuti, $\gamma$ Doradus, red giant, and tidally excited pulsators. We developed a data inspection tool (DIT) that automatically produces a series of plots from the Kepler light curves that allows us to visually identify whether stellar oscillations are present in a given time series. We applied the DIT to the whole Kepler EB database and identified 303 systems whose light curves display oscillations, including 163 new discoveries. A total of 149 stars are flagged as $\delta$ Scuti (100 from this paper), 115 as $\gamma$ Doradus (69 new), 85 as red giants (27 new), and 59 as tidally excited oscillators (29 new). There is some overlap among these groups, as some display several types of oscillations. Despite the likelihood that many of these systems are false positives, for example, when an EB light curve is blended with a pulsator, this catalog gathers a vast sample of systems that are valuable for a better understanding of stellar evolution.
\end{abstract}

Key words. binaries: general - binaries: eclipsing - stars: oscillations - stars: variables: $\delta$ Scuti - asteroseismology - catalogs

\section{Introduction}

It is widely agreed that asteroseismology has become the most reliable way to infer global and internal properties of solar-like stars, from the main sequence (MS) to red-giant (RG) phase, ever since the remarkable success of the space-borne photometers CoRoT, Kepler, and TESS (Baglin et al. 2009; Borucki et al. 2010; Ricker et al. 2015). The simplest and most popular application of asteroseismology consists in comparing the oscillation global properties of a given star to those of the Sun, and retrieving its mass and radius from the asteroseismic scaling relations (Kjeldsen \& Bedding 1995). Since masses and radii lead to ages and distances, solar-like oscillators represent key tracers of the properties of stellar populations. This approach has been used to characterize large samples of stars observed by CoRoT and Kepler, which detected thousands of solar-like oscillation spectra for MS and RG stars (Chaplin \& Miglio 2013; Chiappini et al. 2015). It will also play a central role in the ESA PLATO mission, for which asteroseismic inference is expected to constrain stellar and planetary properties of tens of thousands of systems.

Besides, classical pulsators such as $\gamma$ Doradus $(\gamma$ Dor $), \delta$ Scuti $(\delta$ Sct), $\beta$ Cephei, slowly pulsating B-type stars (SPB), pulsating B stars exhibiting emission lines (Be), or rapidly oscillating Ap stars are also becoming of prime importance. These stars are more massive and hotter than the Sun and display pressure modes (e.g., $\delta$ Sct), gravity modes (e.g., $\gamma$ Dor), or both (hybrid). So far, asteroseismology for $\gamma$ Dor and $\delta$ Sct stars has been hindered due to difficulties in mode identifica- tion, rotational splitting, combination frequencies, mode selection, and mismatch between observed mode spectrum and theoretical predictions. However, very significant progress has been made in the last few years in deciphering the pulsation spectra, providing hope that asteroseismology may be feasible with these stars. Bedding et al. (2015) use échelle diagrams to study $\gamma$ Dor $g$-mode period spacings, and identify sequences of rotationally split multiplets of degrees 1 and 2. Van Reeth et al. (2015a,b) show how to use nonuniform period spacings in Kepler $\gamma$ Dor stars as a seismic diagnostic. García Hernández et al. (2016) report progress in developing a method to use low-order $p$ modes to determine the mean density of $\delta$ Sct stars, and apply it successfully to a CoRoT star. Furthermore, Mirouh et al. (2019) report theoretical studies about period spacing in fast-rotating $\delta$-Sct stars, and Kurtz et al. (2015a) show that complex frequency spectra of $\gamma$ Dor, SPB, and Be stars can be explained by just a few $g$-mode frequencies plus their combination frequencies. It appears that the community is on the verge of a breakthrough in interpreting the frequency spectra of MS pulsators and developing asteroseismic techniques to derive interior structure properties.

Given the importance of asteroseismology for both solarlike stars and classical pulsators, it is of fundamental importance to identify a set of benchmarks with which to refine the stellar models. Such benchmarks are stars whose main physical properties are known with high precision, especially mass, radius, metallicity, and temperature. In recent decades, eclipsing binaries (EBs) have become very popular as benchmarks for stellar physics, as they provide accurate ways to measure masses, 
radii, and distances. It is possible to determine the mass and radius of each component of a double-line spectroscopic binary (SB2) from measurements of eclipse photometry and radial velocities. It is also possible to measure the mass of stars belonging to highly eccentric binary systems nicknamed "heartbeat" (HB) stars because their light curves recall electrocardiograms (e.g., Welsh et al. 2011), hierarchical triple systems (HTs, e.g., Borkovits et al. 2016), and visual binaries (e.g., Marcadon et al. 2018).

Recent space missions based on high-precision photometry drastically changed the number of known EBs, as well as the sensitivity of measurements (e.g., Prša et al. 2011; Debosscher et al. 2011; Coughlin et al. 2011). The original Kepler mission discovered 2,925 $\mathrm{EBs}^{1}$, including over 800 with periods from 10 to 1100 days, over $50 \mathrm{HB}$ systems (e.g., Thompson et al. 2012; Beck et al. 2014; Gaulme et al. 2014; Shporer et al. 2016), and 222 triples displaying eclipse timing variations (ETVs; Borkovits et al. 2016). Beyond Kepler, the CoRoT mission has discovered a few thousand EB systems (Cilia Damiani, priv. comm.), and Kepler's extended mission, K2, has found almost $700 \mathrm{EBs}$ in the first six fields of view (according to the Villanova database).

Regarding classical pulsators, the last 30 years has seen a large amount of research aimed at studying binary systems that include a pulsator (Szatmary 1990). The majority concerns $\delta$-Sct pulsators. We refer the reader to the comprehensive reviews available in Liakos \& Niarchos (2017) and Kahraman Aliçavuş et al. (2017). In brief, there are 199 confirmed cases of binary systems containing at least a $\delta$-Sct pulsator (Liakos \& Niarchos 2017), 87 of which being detached or semidetached EBs, the other being visual, ellipsoidal variables, and spectroscopic binaries. Among the catalog of 199 $\delta$-Sct in binaries, 29 are targets of the NASA original Kepler mission, among which 16 are EBs. Debosscher et al. (2011), who were the first to look for oscillators in EBs observed by Kepler, detected 14 classical pulsators in EBs, of which five are either $\gamma$ Dor or SPB. In addition, Gaulme \& Guzik (2014) reported the identification of eight bona-fide classical pulsators among the Kepler EBs, one including a $\gamma$ Dor and seven $\delta$ Sct. We note that in addition to the EBs, the measurement of fine frequency modulation of classical pulsators observed by Kepler led to the discovery of 341 new binary systems (Shibahashi \& Kurtz 2012; Murphy et al. 2018). These systems are wide binaries - otherwise no fluctuation of mode frequencies would be measurable and are likely not to be eclipsing.

Regarding solar-like oscillators belonging to EBs, all are red giants (RGs) detected by the Kepler mission (Hekker et al. 2010; Gaulme et al. 2013，2014; Beck et al. 2014, 2015; Kuszlewicz et al. 2019, Benbakoura et al., in prep.). So far, eleven wide SB2 EBs including an oscillating RG have been fully characterized with the help of radial-velocity ground-based support (Frandsen et al. 2013; Rawls et al. 2016; Gaulme et al. 2016; Brogaard et al. 2018; Themeß1 et al. 2018) and three more are part of the upcoming paper of Benbakoura et al. (in prep.). We note that an equivalent number of RGs displaying oscillations have been detected in HB systems (Gaulme et al. 2013，2014; Beck et al. 2014，2015; Kuszlewicz et al. 2019), but most do not show eclipses and are single-line spectroscopic binaries (SB1s).

\footnotetext{
1 According to the Villanova Kepler EB catalog, updated on May 6, 2018, which includes 2909 systems (http: //keplerebs . villanova. edu/), and Coughlin et al. (2011) which lists 82 systems among which 16 are not in the Villanova catalog.
}

In the context of the preparation of the new ESA space mission PLATO, where asteroseismology plays a key role, we estimate that it is a good time for an inventory of the stellar pulsators in EBs, a unique class of stellar benchmarks. This motivated us to lead the first systematic search for any stellar pulsators in EBs in the Kepler data. We focus on the original Kepler mission because we can legitimately consider the list of EBs to be complete, whereas the catalog of the extended $\mathrm{K} 2$ mission is not complete. We consider the sample of EBs that is publicly available on the Villanova webpage, in its most recent update (May 2018), and the systems from the Coughlin et al. (2011) paper. The sum of the two catalogs contains 2925 systems, with orbital periods ranging from 0.05 to 1087 days. This global catalog is not in a strict sense a catalog of EBs, as about 600 ellipsoidal binaries are included. Ellipsoidal binaries are noneclipsing tight binaries where the stellar oblateness (ellipsoidal shape) introduces a periodic modulation of the light curve. We focus on $\gamma$ Dor, $\delta$ Sct, and RG oscillators, but we also list all other types of pulsators that we are able to identify, in particular tidal pulsators, and a handful of possible SPBs or white dwarf (WD) pulsators.

Our goal is to decipher whether or not oscillations are detected in the Kepler EBs. We classify the oscillators according to their types, but we do not model each eclipse light curve or attempt to identify the oscillation modes that we detect. The objective is to motivate future in-depth studies of the most interesting cases, which would involve complementary radial velocity (RV) measurements. The paper is organized as follows. We review the main properties of the Villanova EBs and we describe the methods employed to disentangle the stellar pulsations from the eclipse signal in Sect. 2. We then present the detection of 303 systems where pulsations are detected in Sect. 3, including 163 that have been discovered in the present study, before discussing the results in Sect. 4.

\section{Method}

\subsection{Source of information}

Most of the paper is based on the Villanova EB database (e.g., Prša et al. 2011; Slawson et al. 2011; Matijevič et al. 2012; Conroy et al. 2014; Kirk et al. 2016), which contains information about 2909 systems that were extracted with the "eclipsing binaries via artificial intelligence" (EBAI) pipeline (Prša et al. 2008). EBAI is a pipeline based on an artificial neural network that automatically extracts the sum of relative radii $\left(R_{1}+R_{2}\right) / a$, temperature ratio $T_{2} / T_{1}$, orbital argument of the periastron $\omega$, eccentricity $e$ and inclination angle $i$ of a binary system. From the database, it is possible to download estimates of the orbital period, the date of primary eclipse, primary and secondary (if any) eclipse depths, durations (widths), relative separation, eclipse morphology, Kepler magnitude, and effective temperature from up to three different sources (Kepler Input Catalog, Pinsonneault et al. 2012, and Casagrande et al. 2011 catalogs). Regarding data availability and history, the database also indicates whether short cadence (1 min) or long cadence (30 min) data are available, and whether or not publications mentioning a given system exist. In addition, the detection of ETVs - an indicator of interacting triple systems is flagged. We optimized our data processing tools for longcadence data and do not consider possible short-cadence data in this paper.

In Figs. 1 and 2, we present the distribution of the EB sample as a function of orbital period and effective temperature. The shortest orbital period is 0.05 days, the maximum is 1087 days. Ten percent of the stars display a period shorter than 0.33 days 


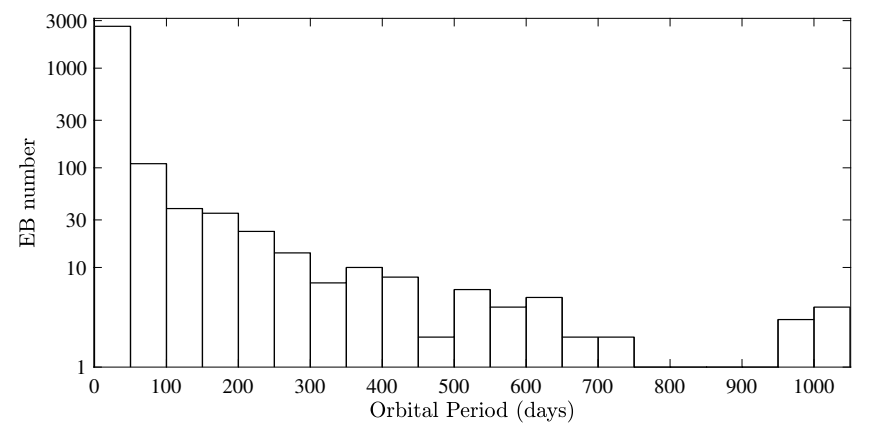

Fig. 1. Orbital period histogram of the Villanova EB systems. The $y$-axis sampling is the logarithm with base ten of the number of systems per step. Out of a total of 2909 systems, 2631 (i.e., 90.4\%) display an orbital period of less than 50 days.

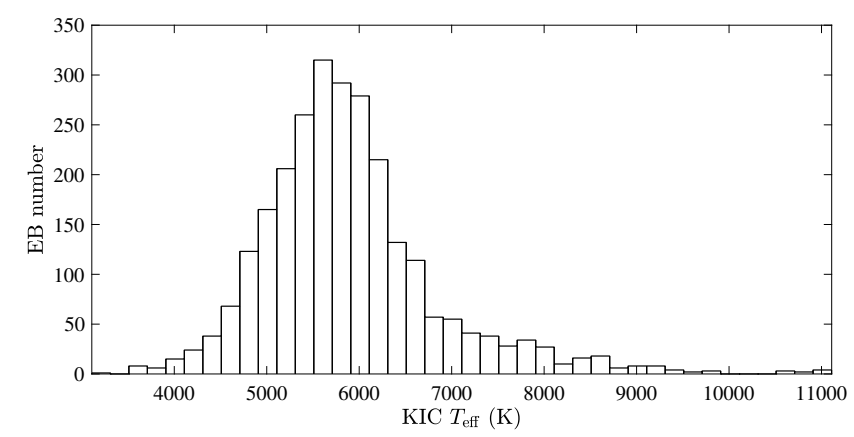

Fig. 2. Effective temperatures of the Villanova EBs. Temperatures come from the Kepler Input Catalog (KIC) and are available for 2625 of the 2909 systems $(90.2 \%)$.

(tenth percentile), and $10 \%$ longer than 43.3 days. The median orbital period is 2.30 days (Fig. 3). Out of the 2625 systems with an estimated effective temperature, the histogram reveals that 545 are in the $[4500,5300] \mathrm{K}$ range, in which we expect $\mathrm{RG}$ oscillators - together with K dwarfs -, and 785 lie in the [60007500] K range where both $\delta$ Sct and $\gamma$ Dor stars are expected.

The eclipse light curves were classified by Matijevič et al. (2012) who introduce the "morphology" parameter $c$, which is a measure of "detachedness" of the system. This parameter is built upon a grid of simulated light curves that range from well-detached binaries to "overcontact" stars. According to these latter authors, all systems with $c<0.5$ are predominantly detached. The range of $c$ for semidetached systems broadly lies in the $0.5<c<0.7$ range. Overcontact systems dominate the $0.7<c<0.8$ region, after which a mixture of ellipsoidal variables and systems with uncertain classification sets in, including many HB systems. Figure 3 shows the histogram of the typology of the systems as a function of orbital period based on this rough classification. We note that among the 2909 systems, 175 are not classified. Out of the 2734 systems that are classified, 1431 (52.3\%) are detached systems, 413 are semidetached (15.1\%), $290(10.6 \%)$ contact binaries, and $599(21.9 \%)$ are classified as ellipsoidal or miscellaneous. It is very likely that most ellipsoidal binaries with periods shorter than one day are either contact or semidetached systems. It is also worth noting that detached systems dominate the sample starting from the median period ( $P \geq 2.30$ day).

Even though most of the paper is based on the Villanova database, a handful of systems that were published by Coughlin et al. (2011) have never been included in the Villanova database. We count 16 of these systems and include them in our analysis. We also add the RG in an eclipsing HB system recently studied by Kuszlewicz et al. (2019), which is not listed

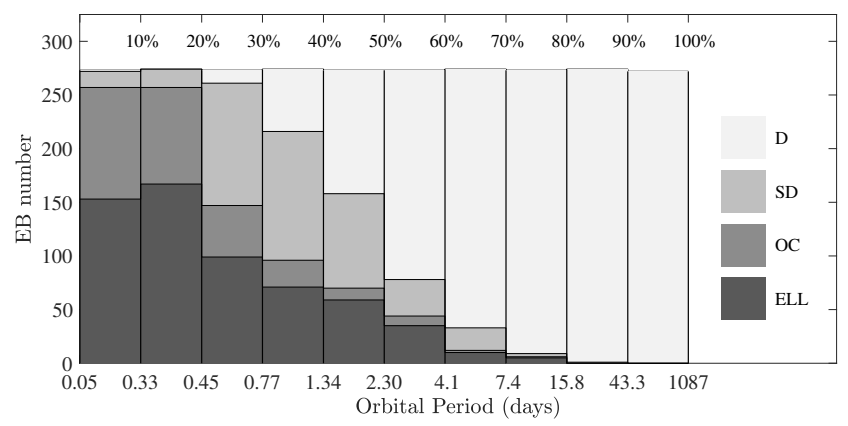

Fig. 3. Eclipse light curve classification according to Matijevič et al. (2012) as a function of orbital period. The size of each bloc is adjusted to include $10 \%$ of the total number of systems that were classified. The total number of classified systems is 2734 and each chunk consists of $273 \pm 1$ systems. The median orbital period is 2.30 days, while the 10 and 90 percentiles are 0.33 and 43.3 days, respectively. The labels " $D$ ", "SD", "OC", and "ELL" refer to detached, semidetached, overcontact and ellipsoidal systems. A total of 1431 systems are D, 413 are SD, 290 are OC, and 599 are ELL, i.e., a total of 2135 systems are classified as EBs.

in the Villanova catalog. We performed our study with Kepler public light curves available on the Mikulski Archive for Space Telescopes (MAST) ${ }^{2}$. We work with both the Simple Aperture Photometry (SAP, i.e., raw data) and the Pre-search Data Conditioning Simple Aperture Photometry (PDCSAP) light curves. The latter consist of time series that were corrected for discontinuities, systematic errors and excess flux due to aperture crowding (Twicken et al. 2010). We note that we do not seek complementary information from the Gaia data release 2 cata$\log$ because binary stars are not included, and if some are, their parameters may be biased.

\subsection{Disentangling eclipses, surface activity, and oscillations}

Searching for pulsations in EBs entails removing the photometric variability caused by binarity: mainly eclipses and phase effects. The methods we use are described in Gaulme et al. $(2013,2014,2016)$ and here we provide a summary.

The major challenge in concatenating light curves is to ensure photometric continuity before and after each interruption. The main cause of photometric jumps from quarter to quarter is the fact that the Kepler telescope rotated four times a year, which implied that a given star would fall on four different chips. However, the pointing was fine enough that a star repeatedly covered the same group of pixels every four quarters. Light curves are obtained by adding the pixels of the masks that were designed for every star of the field of view. For a given star, a mask was designed for each of Kepler's positions. Because of the photo response nonuniformity (PRNU) of the pixels and the changing size of the masks, the recorded flux changes. Both PRNU and varying mask areas lead to flux discontinuity that should be adjusted in a multiplicative way. The first correction we apply is therefore a normalization that turns the photoelectric counts into relative flux, by dividing the light curve of each quarter by its average. A median is actually more appropriate than a mean as outliers and large photometric jumps can bias the mean. If photometric variations would only be generated by PRNU and masks, this process should be enough. We note that this is only true for systems where no stellar activity is measurable if we exclude the effect of the differential velocity aberration.

2 http://archive.stsci. edu/kepler/ 
Issues arise with the systems that display strong pseudoperiodic luminosity fluctuations. For those, the average (or median) over a quarter is biased by the fact that the number of pseudo periods is too small to be averaged out. Therefore, the median is not a perfect estimator of the mean photometry. This is an intrinsic limitation of the light curve photometric accuracy. In such cases, jumps remain with amplitudes within a few percent. Given that the remaining jumps are caused by a biased normalization, the second layer of adjustment to be applied should still be done in a multiplicative way. However, this is not possible in practice because none of the quarters can be considered as an absolute reference. The only corrections we may apply are additive, to ensure a smooth aspect of the light curve and to minimize their effects in the Fourier domain.

We employ two ways to smooth the remaining discontinuities once quarters are divided by their median. When a gap is short with respect to the photometric variability timescale, each side of the gap is adjusted accordingly. When a gap is longer than the variability period, we simply adjust the photometry with the difference of the means of each chunk surrounding the gap. Once the complete time series is leveled and concatenated, a linear fit is subtracted from it to take into account the decreasing instrumental sensitivity. Finally, when working with SAP light curves, we compensate for the differential velocity aberration - the motion of the target across a fixed aperture smaller than the point spread function - caused by the pixel scale breathing along the orbit of the satellite (372.5 days), whose peak-to-peak amplitude ranges from $0.5 \%$ to $\sim 10 \%$. This is done by subtracting from each light curve a 372.5-day period sine fitting and a first harmonic, which is enough to reduce its amplitude to less than $0.5 \%$.

We search for stellar pulsations in the power density spectra of the light curves. To minimize the effects of the incomplete duty cycle, we perform gap fillings and make use of the fast Fourier transform. All short gaps (only several missing points) are interpolated with a second-order polynomial estimated from the nearby data points. Long gaps are filled with zeros. To reduce the impact of abrupt discontinuities around long gaps, the edges of each section in between gaps are apodized with a cosine function. This is particularly important when significant variability is detected.

For well-detached systems, in which the time spent during eclipses is less than $20 \%$ of the time, we remove the data corresponding to the eclipses and bridge them with a secondorder polynomial constrained by the surrounding data. In cases where time spent during eclipses is more than $20 \%$, we prefer to fold the light curve on the orbital period and subtract the average signal from each orbital period. Another option could have been to model the eclipse light curve with a fitting routine, such as PHOEBE (Prša 2018) or JKTEBOP (Southworth 2013). However, the advantage of detrending the light curves with a mean folded light curve is that the signal does not have to be modeled, which represents a huge amount of work for a sample of 3000 targets. In addition, residuals of a light curve minus a model are often significant enough to alter the signal. Still there is a big drawback when opting to subtract the folded light curve: because of pointing jitter and PRNU, the amplitude of eclipses may vary from quarter to quarter, or even during a quarter. Consequently, there are significant residuals in the light curves, and a signature of the eclipse signal is still quite visible. In such cases, we then remove all harmonics of the orbital period from the Fourier spectra. The latter method is not very sophisticated but can still help in detecting classical pulsator oscillations, as illustrated in Fig. 5 (middle panel, gray vs. black curve).

\subsection{Data inspection tool}

We developed a quick-look data-inspection tool (DIT) that helps the user to classify a system at a glance (Fig. 4$)^{3}$. For each star, it is composed of a page divided into a series of ten plots. First, the original Kepler light curves (SAP and PDCSAP) are displayed to look for any major issue regarding the data (panel a). This is useful in the case where a quarter or two are outliers with respect to the others and need to be manually removed before reprocessing the light curve. In a second panel b, the three types of light curves described in the previous section (variability, eclipse, oscillation types) are overplotted to make sure that the disentangling is correct. Specifically, the user checks whether discontinuities are still present in the oscillation-optimized light curve. A fold of the complete light curve is also a good indicator of any misestimate of the orbital period, or of the presence of a third body eclipsing or causing ETV (panel c). A zoom on each eclipse is also displayed for refining this analysis for long orbits, where the eclipses represent less than $10 \%$ of the period (panels $\mathrm{d}$ and e). We also plot a zoom on a relatively short range (20 days) at a random location, to help the user visually identify slow pulsations in the time domain, such as $\gamma$-Dor or tidally induced (panel f).

The last four plots are the power spectral density (PSD) in $\log -\log$ scale to identify surface activity (typical of solar-like stars) and outstanding peaks indicating oscillations (panel g). It is also useful to check the quality of the background fitting that is performed to whiten the PSD. The background fitting is done following the prescription of Kallinger et al. (2014) for solar-like oscillators. The next plot is the square root of the previous one, that is, the Fourier transform module, which is more appropriate to identify pulsators with a large variety of oscillation amplitudes, such as $\delta$-Scuti (panel h). The last two plots are more focused on the search for solar-like oscillators, as they display the envelope of the autocorrelation of the time series (EACF; see Mosser \& Appourchaux 2009), which is usually considered the best performing tool to detect solar-like oscillations and measure the large frequency spacing $\Delta v$, even in low signal-to-noise-ratio $(\mathrm{S} / \mathrm{N})$ conditions (panel i). The last panel is an échelle diagram of the PSD based on the $\Delta v$ deduced from the EACF computation (panel j).

In addition to the plots, the title includes the orbital period, the data sampling (long or short cadence), the light-curve type (SAP, PDCSAP), and the effective temperature. The latter is particularly useful when oscillations are detected. For example, an effective temperature of $4900 \mathrm{~K}$ is compatible with the detection of RG oscillations, and a temperature of $7000 \mathrm{~K}$ with a $\delta$ Scuti. We are aware that published temperatures may be inaccurate, or often not available, but it is valuable support information.

\section{Results}

We ran the DIT on all of the targets from the 2018 update of the Villanova catalog, except for the RGs in EB or HB systems published by Hekker et al. (2010), Gaulme et al. (2013, 2016), Beck et al. (2014, 2015), and the forthcoming paper by Benbakoura et al. (in prep.). Indeed, these systems were already well characterized, and first author Gaulme had already processed them. Regarding the classical pulsators, we were not sure about the exact number of systems already known and we did not exclude them a priori from the analysis. The total amount of stars for which we applied the DIT pipeline and that we visually inspected is 2875 out of a total of 2925 .

\footnotetext{
3 The ultimate goal of the DIT is to be public, but it is still under development and more time is required.
} 

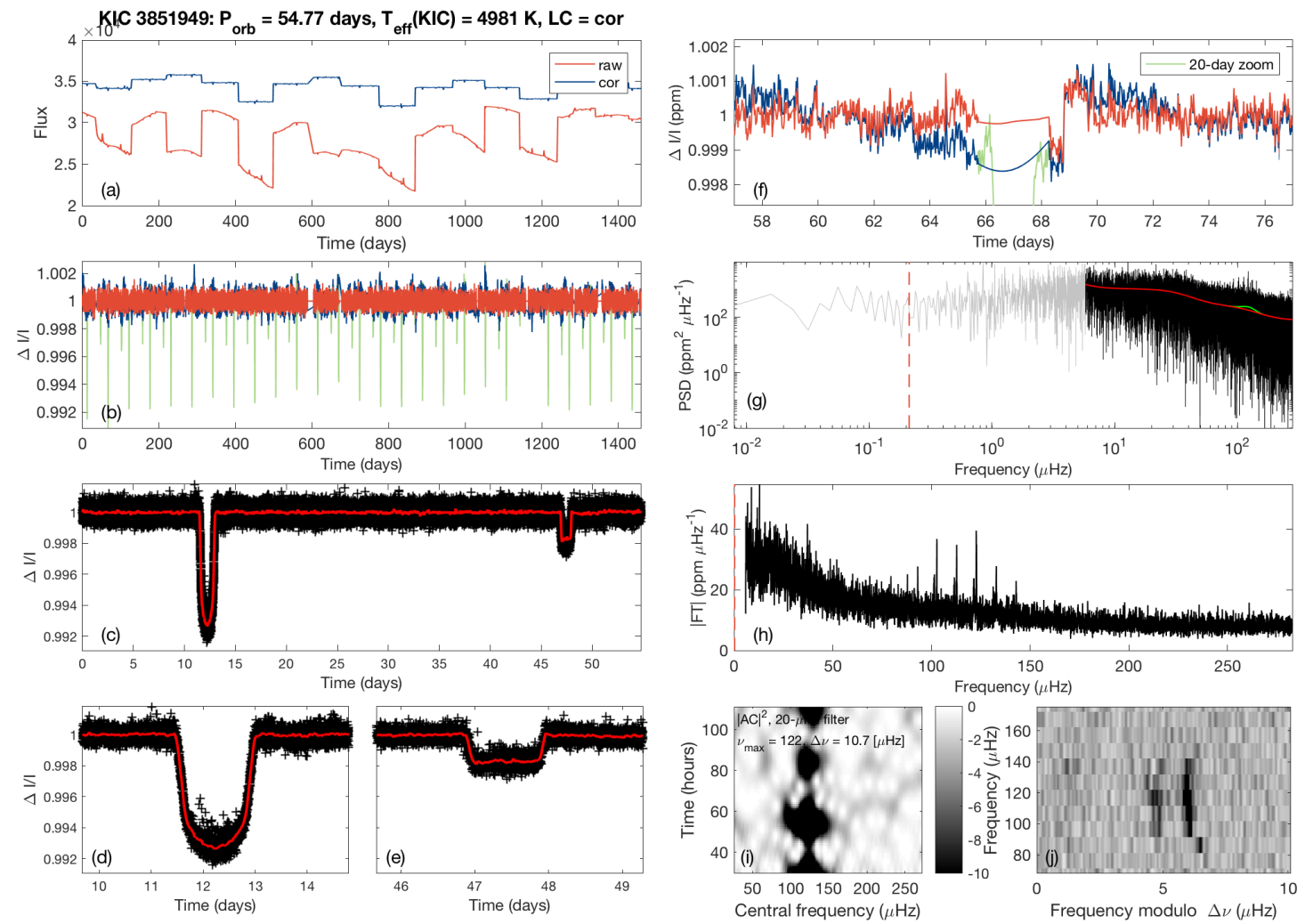

Fig. 4. Data inspection tool applied to the EB system KIC 3851949, which includes a solar-like oscillator with $v_{\max } \approx 120 \mu \mathrm{Hz}$ and depleted $\ell=1$ modes. Left column, from top to bottom, panel a: Kepler light curves as a function of time (days), where "raw" stands for SAP and "cor" for PDCSAP. Panel $b$ : light curves expressed in relative flux, where the blue line contains the stellar activity and oscillations (eclipses clipped out), the green line is the eclipse signal (activity filtered out), and the red curve is optimized for oscillation searches (activity and eclipses filtered out). Panel $c$ : eclipse light curve folded on the orbital period. Panels $d$ and $e$ : zooms of the folded light curves around the eclipses. Right column, from top to bottom, panel $f$ : zoom of the light curve over 20 days. Panel $g: \log -\log$ scale display of the power spectral density of the time series expressed in $\mathrm{ppm}^{2} \mu \mathrm{Hz}^{-1}$ as a function of frequency $(\mu \mathrm{Hz})$. Panel $h$ : amplitude Fourier spectrum of the time series as a function of frequency. Panel $i$ : envelope of the autocorrelation function (EACF) as a function of frequency and time. Panel $j$ : échelle diagram associated with the large frequency spacing automatically determined from the EACF plot. The $x$-axis is the frequency modulo the large frequency spacing (i.e., from 0 to $\Delta v$ ), and the $y$-axis is frequency.
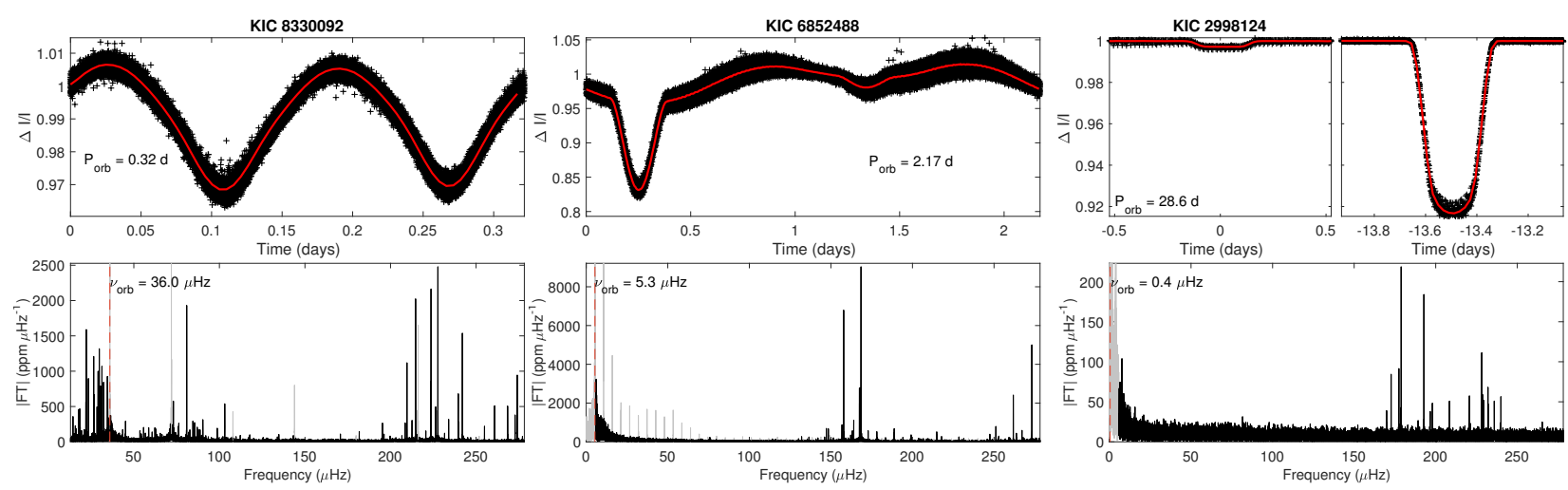

Fig. 5. Examples of classical pulsators observed in the light curves of binary systems. Left panel: OC binary (KIC 8330092) with an orbit of 0.32 days displaying $\gamma$ Dor and maybe Rossby modes at $v \lesssim 50 \mu \mathrm{Hz}$ and clear $\delta$ Sct oscillations above that. Middle panel: short-period detached system KIC 6852488 in which $\delta$ Sct oscillations are clearly visible at $v \gtrsim 120 \mu \mathrm{Hz}$. The asymmetrical dispersion of points around the rebinned folded light curve is caused by the presence of spots that modulate the photometric variations as a function of time. Right panel: "long"-period detached EB KIC 2998124 displaying $\delta$ Sct oscillations at $v \gtrsim 170 \mu \mathrm{Hz}$. Vertical dashed red lines indicate the orbital frequency $v_{\mathrm{orb}} \equiv P_{\mathrm{orb}}^{-1}$. For left and middle plots, the gray curve is the modulus of the Fourier transform prior to removing harmonics of the orbital period.

We first inspected every light curve to check whether orbital parameters were off. In 100 cases, we had to modify the ephemeris published on the Villanova database; many were small inaccuracies regarding eclipse timing and duration. Also, for 36 systems, only primary eclipses were detected. Thanks to the visual inspection of the light curves folded on the orbital period, we identified secondary eclipses for 36 new systems.

Even though it can sometimes be difficult to distinguish the detection of oscillations from imperfect light curve cleaning, we iteratively converged to the detection of pulsations in 303 out 
of the 2925 light curves, that is, in about $10.4 \%$ of the cases. To do so, first author Gaulme produced a first screening by inspecting all of the DIT files, and selected about 350 of them. He attempted a preliminary classification according to pulsator type. Co-author Guzik then reviewed all of them and commented on each case with no instruction from Gaulme. Subsequently, Gaulme led a systematic search of every target on a web search engine by entering "KIC" and the KIC number of each star, one by one, with quotes and without quotes. This ended up being more efficient than using publication search tools such as NASA ADS or SIMBAD. The result of this search is certainly not fully exhaustive but is relatively complete. In total, we found 187 systems to have been studied already in one way or another in a peer-review paper or a conference proceeding. The level of study was highly variable, from simple notes in large tables containing many Kepler targets to dedicated papers about single binary systems. Having 187 systems previously cited in the literature does not mean that all were characterized both as binary and pulsators. Indeed, many were studied as double or triple systems but there was no mention of any stellar pulsation. Only 140 systems had already been reported as being part of a multiple-star system and containing a stellar pulsator. The main result of the present paper is that we have identified 163 new pulsating stars in multiple star systems. This represents $54 \%$ of the 303 stellar pulsators identified in the Kepler EB catalogs, and an increase of $116 \%$ of the known stellar pulsators in EBs among the Kepler data.

Not all of the pulsators we list are actual pulsators in multiple-star systems. Many are false positives, that is, pulsators that are either aligned (within the point spread function or pixel) with an EB or bright stellar pulsators whose light leaks into the considered EB mask. Disentangling those is a huge work that goes well beyond the scope of the present work. To do so, one should first check the Kepler imagettes and check whether the maximum intensity matches the maximum amplitude of the oscillations and the maximum depth of the eclipses. Such an approach was used by Gaulme et al. (2013) for 70 RG candidates in EBs. The second step would consist of consulting existing RV measurements or acquiring new ones. However, we can get a very rough idea of the likelihood that a pulsator belongs to a given binary system. For example, if we detect very clear oscillation peaks in the Fourier domain, while the photometric variability associated with the eclipses is significantly less than $1 \%$, we can assume that the pulsator is not a member of the EB. Nevertheless, it could still be a triple system. Another example, a $10 R_{\odot} \mathrm{RG}$ associated with a binary system that orbits in less than 5 days is very unlikely. Indeed, in such systems, stars are synchronized and the rotation rate of the star could make it disintegrate, depending on the orbital parameters. For RGs specifically, Gaulme et al. (2013) studied the question and used this type of consideration to detect many false positive RGs in EBs.

Our results are displayed in Table A.1, which includes the 303 systems where we are confident to have detected oscillations, plus the system identified by Kuszlewicz et al. (2019). The table is sorted by increasing KIC number; it is optimized to occupy as little room as possible and cannot include all the existing information. We therefore refer to the Villanova catalog and the Coughlin et al. (2011) paper for complete details of orbital parameters. We only list the main properties an observer would need to decide which target to study and observe: effective temperature, Kepler magnitude, orbital period, deepest eclipse depth, phase separation in between primary and secondary eclipses, ratio of eclipse durations, sum of eclipse durations relative to orbital period, and pulsator type. We indicate the number of eclipses per orbital period, which dictates whether it is a true EB or an ellipsoidal-variation binary, or even a HB star. We also list the references we have identified and systems marked with "Y" are those for which this is the first they have been identified as both a pulsator and a binary. Some notes were added to the last column to point out random relevant information.

\section{Discussion}

In this section, we highlight the main findings of the current study and list actions that could be taken based on this sample for future studies.

\subsection{Classical pulsators}

Classical pulsators represent the majority of the pulsators in EBs, as we expect from the effective temperature histogram (see Sect. 2.1). A total of $190 \gamma$ Dor, $\delta$ Sct, or hybrids are identified, including 122 that were previously unknown. There is more uncertainty regarding the detection of $\gamma$ Dor oscillations with respect to $\delta$ Sct because of the frequency range that often overlaps harmonics of the orbital frequency. Due to the large amount of data, we did not look for regular period spacings or whatever type of pattern which would indicate a $\gamma$ Dor pulsator. We simply flagged as $\gamma$ Dor pulsators any stars that display pulsation frequencies of less than $\sim 50 \mu \mathrm{Hz}(\sim 5 \mathrm{c} / \mathrm{d})$ and possibly typical $\gamma$ Dor features in the time domain (e.g., Kurtz et al. 2015a). In addition, any information about effective temperature helped us to assess $\gamma$ Dor pulsators. We flagged $\delta$ Sct stars whose oscillation spectra resembled those of typical $\delta$ Sct (e.g., Baglin et al. 1973; Balona et al. 2015), with frequencies larger than $50 \mu \mathrm{Hz}$. However, in some cases, oscillation spectra are sparse (few peaks), and there could be some doubt surrounding tidally excited oscillations when peaks are regularly spaced. In some other cases, oscillation spectra closely resemble those of a $\delta$ Sct but the stellar effective temperatures are slightly too hot. In these cases we wrote a note in the table, but we keep in mind that effective temperatures from the KIC may be inaccurate, especially for close binary systems. We display three examples of $\gamma$ Dor and $\delta$ Sct pulsators in Fig. 5.

A rather surprising finding of this study is the relatively large number of $\gamma$ Dor and/or $\delta$ Sct pulsators in very-short-period systems. A total of 13 systems with orbital periods of less than 0.5 days display clear oscillations. Some could either be false positives or triple systems. Indeed, three of them are triple systems as ETVs were measured and published in previous articles (Gaulme et al. 2013; Borkovits et al. 2016). However, a more detailed investigation would be worthwhile to decipher whether the ten remaining systems are false positives, triples, or genuine EBs.

The first panel of Fig. 5 is an example of our short-period classical pulsators with the 0.32-day OC binary KIC 8330092. The oscillation spectrum is very clear, with a low-frequency part which we assume to be $\gamma$ Dor and higher frequencies that are typical of $\delta$ Sct. In addition, the crowded aspect of the low-frequency part could be partially composed of Rossby modes as proposed by Saio et al. (2018). In the specific case of KIC 8330092, since the system is an OC, its rotation period is equal to its orbital period. From the light curve, the system seems to be composed of two similar stars (similar eclipse depth and shape), which we assume to be A0- to F5-type stars, that is, with radii and masses of about $1.4 R_{\odot}$ and $1.4 M_{\odot}$. Within such an assumption $\left(M_{1}=M_{2}, R_{1}=R_{2}\right)$, the rotational velocity at the equator is $V_{\mathrm{eq}} \approx 220 \mathrm{~km} \mathrm{~s}^{-1}$, whereas the escape velocity from 
the gravitational field is $V_{\mathrm{esc}} \approx 730 \mathrm{~km} \mathrm{~s}^{-1}$, which means that such a star is far from disintegrating due to fast rotation. Moreover, still within the same assumptions, Kepler's third law indicates that the semi-major axis of the system would be $a \approx 2.8 R_{\odot}$. These numbers are very rough estimates, but they indicate that a star such as a $\delta$ Sct can exist in such a tight contact binary.

Discovering classical pulsators in short-period binary systems is not something new per se. Indeed, several papers have reported detections of $\delta$ Sct or $\gamma$ Dor pulsators in tight binary systems. Here, we mention a few examples. Aerts et al. (2002) report the detection of $\delta$ Sct pulsations in the 1.15-day orbit ellipsoidal binary XX Pyx. Dal \& Sipahi (2013) report the detection of $\delta$ Sct oscillations in the 0.69-day orbit ellipsoidal binary V1464 Aql, and Sipahi \& Dal (2014) the detection of $\gamma$ Dor oscillations in the 0.93-day orbit close binary systems. Zhang et al. (2015) reported the detection of $\delta$ Sct oscillations in a component of the 0.65 -day orbit near-contact binary V392 Ori. From the present study, the ten systems displaying $\gamma$ Dor and/or $\delta$ Sct with $P_{\text {orb }}<0.5$ days are anomalous as to our knowledge no oscillations have been detected so far in such short-period systems. In other words, what is new here is the relative amount of short-period binaries among the oscillators (7\% with $P_{\text {orb }}<0.5 \mathrm{~d}$ ) but it is also the first time that we identify pulsators in systems with periods of less than 0.65 days. Although some may be false positives, it is still very likely that we have identified the $\delta$ Sct in a binary system with the shortest orbit ever recorded.

Beyond short-period systems and thanks to the large amount of $\delta$ Sct that we have, we can test observations that have been made in previous studies. As presented first in Soydugan et al. (2006) then confirmed on a larger sample by Liakos \& Niarchos (2017), there exists a correlation between the pulsation frequency and the orbital period. For systems with $P_{\text {orb }}$ less than a threshold located in between 13 and 40 days, the dominant $\delta$ Sct pulsation tends to increase with decreasing orbital period (see Fig. 4 in Liakos \& Niarchos 2017). In Fig. 6, we test whether we find the same trend from the 149 possible $\delta$ Sct that we identify. For all systems flagged as possible $\delta$ Sct pulsators, we measured the frequency and height of the largest peak belonging to the $\delta$ Sct domain, that is, by excluding the $\gamma$ Dor region for the hybrids. There is no obvious trend visible from our sample. In the most populated part of the diagram, we note a small trend however: for $0.6<P_{\text {orb }}<1.4 \mathrm{~d}$ the most likely pulsation frequency is $20 \mathrm{c} / \mathrm{d}$, for $1.4<P_{\text {orb }}<2.1 \mathrm{~d}$ this is $16 \mathrm{c} / \mathrm{d}$, and for $2.1<P_{\text {orb }}<3.2 \mathrm{~d}$ it is $13.5 \mathrm{c} / \mathrm{d}$.

Several reasons may be responsible for not confirming the correlation between the pulsation frequency and the orbital period. Firstly, we have no way of knowing which systems are false-positive $\delta$ Sct binaries, while Liakos \& Niarchos (2017) used systems that were well characterized. Secondly, we have not looked at the possible short-cadence data for part of our systems. Therefore, our maximum frequency available is the Kepler long-cadence Nyquist frequency $(\simeq 283 \mu \mathrm{Hz})$. This means that a fraction of the frequencies that we present are likely to be aliases of higher frequencies. To emphasize this bias and help the comparison, we kept the $y$-axis boundaries to be the same as that in Liakos \& Niarchos (2017) and we plotted the logarithm in base 10 of the pulsation and orbital periods. Thirdly, the Kepler sample lacks long-period systems, which prevents us from having a view on long-orbit systems ( $8 \delta$ Sct with $P_{\text {orb }}>30$ days). Once the sample we list here is fully characterized, in particular with the help of complementary RV measurements, it will be possible to lead such a study.

In addition to the pulsation-dominant frequency, we tried to see whether binarity suppresses pulsation of $\mathrm{A} / \mathrm{F}$ stars, as was
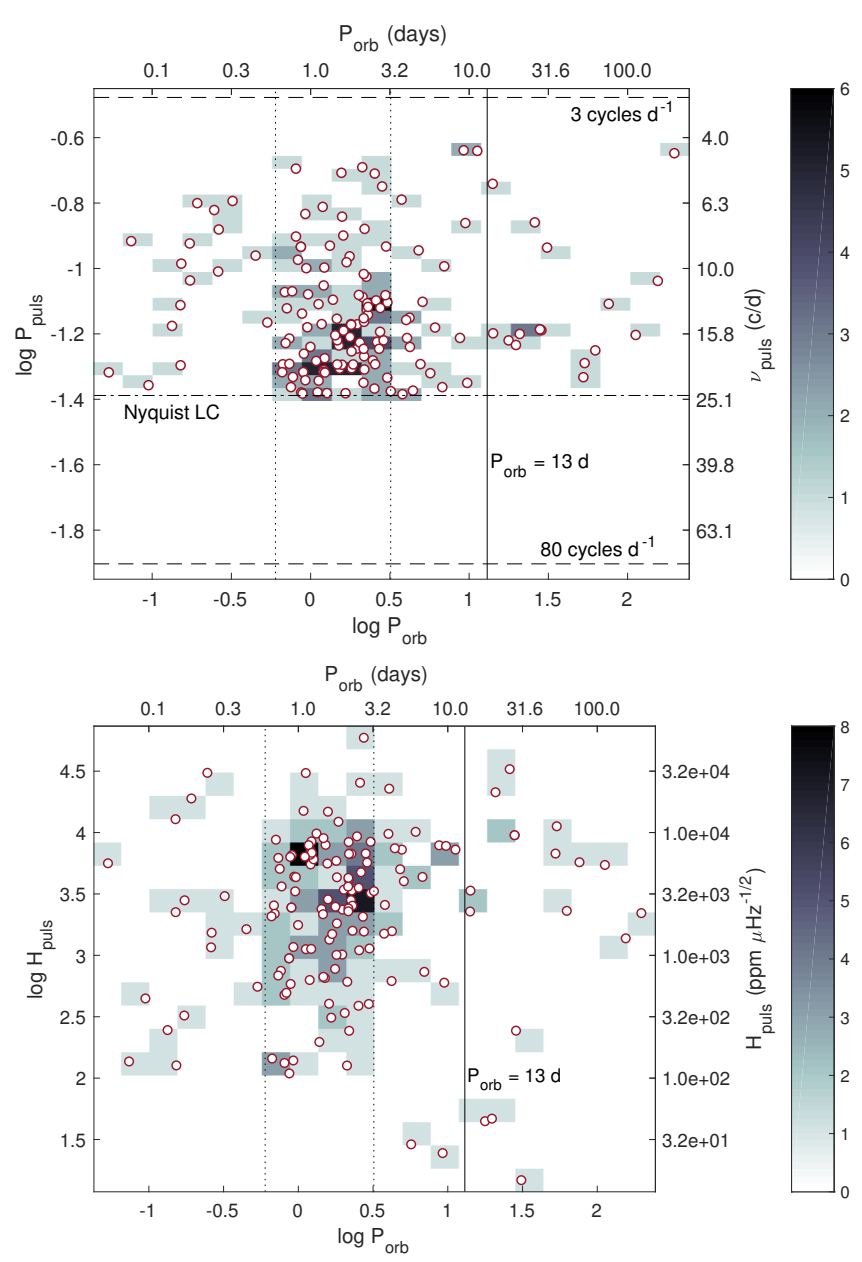

Fig. 6. Pulsation properties of the $148 \delta$ Sct EB candidates identified in this work. Top: pulsation periods of the highest peaks of the oscillation spectra $P_{\text {puls }}$ as a function of orbital periods $P_{\text {orb }}$. Bottom and left $x$ - and $y$-axes are in the same units as in Liakos \& Niarchos (2017). As in this latter paper, the vertical line indicates a 13-day orbital period, while the horizontal lines indicate pulsation frequencies of 3 and 80 cycles per day (c/d), respectively. Top and right $x$ - and $y$-axes are the same in days for $P_{\text {orb }}$ and in frequency for pulsations. Bottom: representation of the height of the highest peak per $\delta$ Sct spectrum as a function of $P_{\text {orb }}$. In both panels the color scale indicates the number of systems per square, to help the reader look for trends, such as possible clustering. The two vertical dotted lines indicate orbital periods from 0.6 to 3.2 days.

observed by Gaulme et al. (2014) for RGs in close binary systems. We looked for a dependence of the pulsation amplitude as a function of orbital frequency. The bottom panel of Fig. 6 does not seem to show any correlation. More generally it would be interesting to see whether the fraction of binaries that do not pulsate is different from the fraction of stars in the instability strip that do not pulsate. Answering this question is tricky as the boundaries of the $\mathrm{A} / \mathrm{F}$ pulsators are not particularly clear due to a lack of precision in the stellar parameters.

\subsection{Solar-like oscillators}

As indicated in the introduction, all solar-like oscillators are RGs. We detect $85 \mathrm{RG}$ oscillation spectra among all the binaries listed in the Villanova catalog and the Coughlin et al. (2011) table. Among those, 27 are newly identified as being both oscillating RGs and binary (eclipsing, ellipsoidal, or HB). As mentioned earlier, a large fraction of them must be either false 

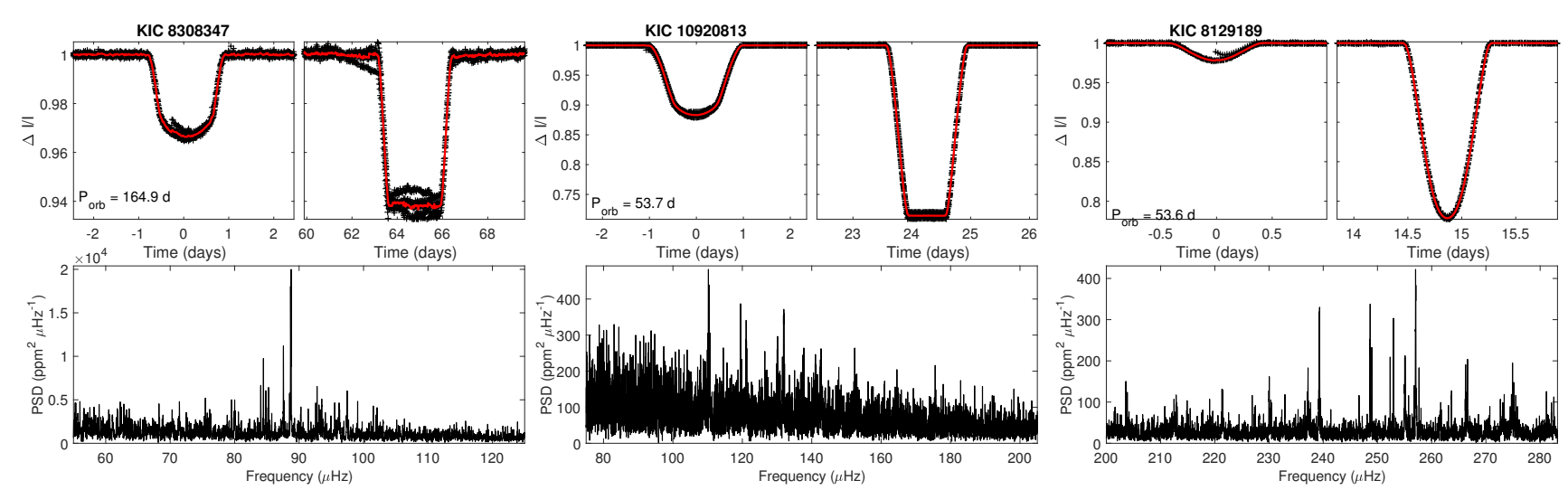

Fig. 7. Bona fide RGs/EBs discovered in the present work that are possibly SB2. Top panels: Kepler light curves folded over the orbital period (black markers), with a rebinned version of it in 30-min steps (red line). Bottom panels: PSD as a function of frequency of the Kepler light curves after eclipse removal - centered around the RG oscillation modes. Power spectral density was smoothed with a triangular weighted moving average (three bins wide at half maximum) to highlight the oscillation mode visibility.

positives or triple systems. Even though only RV measurements and close-up imaging could allow us to verify their nature, we already have a general idea of the nature of these systems (false positive, binary, triple).

First of all, by following the conclusions of Gaulme et al. (2013), we are suspicious of RGs associated with a system with an orbital period of less than 10 days, especially if it is oscillating (Gaulme et al. 2014). As an example, so far, the shortest oscillating RG in a binary system is the EB KIC 8702921 with a 19.38-day orbit (Gaulme et al. 2016). Second, the shape of eclipses gives a strong indication of the nature of the system. Since the red giant branch (RGB) represents a short period compared to the overall star lifetime, both components are relatively unlikely to be at the same evolutionary stage. Therefore, as observed in practice, the large majority of RGs that belong to binary systems are on the RGB and are composed of a MS star and an evolved star (e.g., Gaulme et al. 2013; Beck et al. 2014). Therefore, one of the two stars is much smaller than the other, which means that one eclipse displays a flat bottom, while the other has a round shape caused by the limb darkening law of the RG star. Systems composed of a white dwarf and a MS star produce similar light curves to those composed of an M-dwarf and a subgiant; occasionally a hot Jupiter transiting a faint star can also produce this kind of light curve as well, provided the stellar light reflected by the planet is enough to cause a secondary transit. The system effective temperature, even if possibly biased, is complementary information that can help confirm that a system hosts an RG.

Beyond eclipsing systems, many "false positive" RG/EBs are actually HT systems, that is, systems where a close EB orbits an RG. In rare cases, the close EB also eclipses the RG (Derekas et al. 2011), but in general this is not the case (e.g., Gaulme et al. 2013). In this kind of system, the RG tends to cause ETVs. The detection of ETVs is a strong indication that the RG belongs to the triple system. Overall, when no ETVs are measured and the orbital period is too short to be an EB including an RG component, we classify a target as "likely FP or triple". We note that even if we do not detect ETVs, we cannot totally exclude a triple system, as ETVs may exist in wide hierarchical triple systems at a level that is not detectable (low ETV amplitude, slow variations).

Based on these criteria, we estimate that 15 out of the 27 systems flagged as displaying RG oscillations are bona fide pulsators in multiple-star systems. Specifically, ten are EBs, one is an HB with no eclipse, and four are HTs. Another system is a possible RG/EB (KIC 10858117), but the oscillation S/N is too poor for a robust conclusion on whether or not there are indeed RG oscillations in the Fourier spectrum of the time series.

As mentioned above, solar-like oscillators in EBs are unique targets for testing and calibrating asteroseismology. To this aim, individual masses and radii are needed, which implies that the EB systems must be double-lined spectroscopic binaries (SB2) to be considered as asteroseismic test benches. Given that most systems are composed of an MS star with an RG, the flux contrast in between both components is large; the companion usually accounts for a maximum of $10 \%$ of the total flux. In practice, for a system to be SB2, the companion star must be hotter than the RG, that is, a G- or F-type dwarf (e.g., Gaulme et al. 2016; Hełminiak et al. 2016, 2017a). With the present work, we can estimate how many $\mathrm{RG} / \mathrm{EB} / \mathrm{SB} 2$ are present in total in the Kepler sample. So far, $11 \mathrm{RB} / \mathrm{EB} / \mathrm{SB} 2 \mathrm{~s}$ have been identified and studied with complementary RV data (Frandsen et al. 2013; Rawls et al. 2016; Gaulme et al. 2016; Hełminiak et al. 2015, 2016; Brogaard et al. 2018; Themeß1 et al. 2018). In the forthcoming paper by Benbakoura et al. (in prep.), three more systems are identified. Furthermore, among those three, one shows no secondary eclipses due to a large eccentricity and a grazing primary, and only masses can be estimated. We are therefore at $14 \mathrm{RG} / \mathrm{EB} / \mathrm{SB} 2$. In addition, one system from the Gaulme et al. (2016) paper (KIC 8054233) which has a 1058-day orbit was classified as SB1, but it could be an SB2 - higher-S/N observations are needed to make a robust conclusion. Hitherto, the total amount is thus 15 at best.

Among the new systems identified in this paper, only KICs 8308347 and 10920813, with orbital periods of 165 and 54 days, respectively, show evidence that the temperature of the companion is higher than that of the giant (" $T_{2}>T_{1}$ " in Table A.1). Another case, KIC 8129189 (54-day period), displays deep partial eclipses and a clear oscillation signal with $\nu_{\max } \approx 250 \mu \mathrm{Hz}$, which indicates that it could be composed of a small RG (for which oscillations are detected) and a smaller RG or a subgiant star. This latter could also be an SB2. The case of KIC 10491544 displays the same kind of eclipses, namely partial; with $T_{2} \sim T_{1}$, an RG of about $6.6 \pm 0.3 R_{\odot}$, and an orbit of 23 days could be a bona fide RG. However, their depths vary from quarter to quarter in between 2 and $5 \%$ and the $\mathrm{S} / \mathrm{N}$ on oscillation measurements is very high, whereas it has been observed that modes are greatly depleted in such short-orbit systems (Gaulme et al. 2014). This latter could be a false positive. Overall, it appears that the number of $\mathrm{RG} / \mathrm{EB} / \mathrm{SB} 2 \mathrm{~s}$ from the 

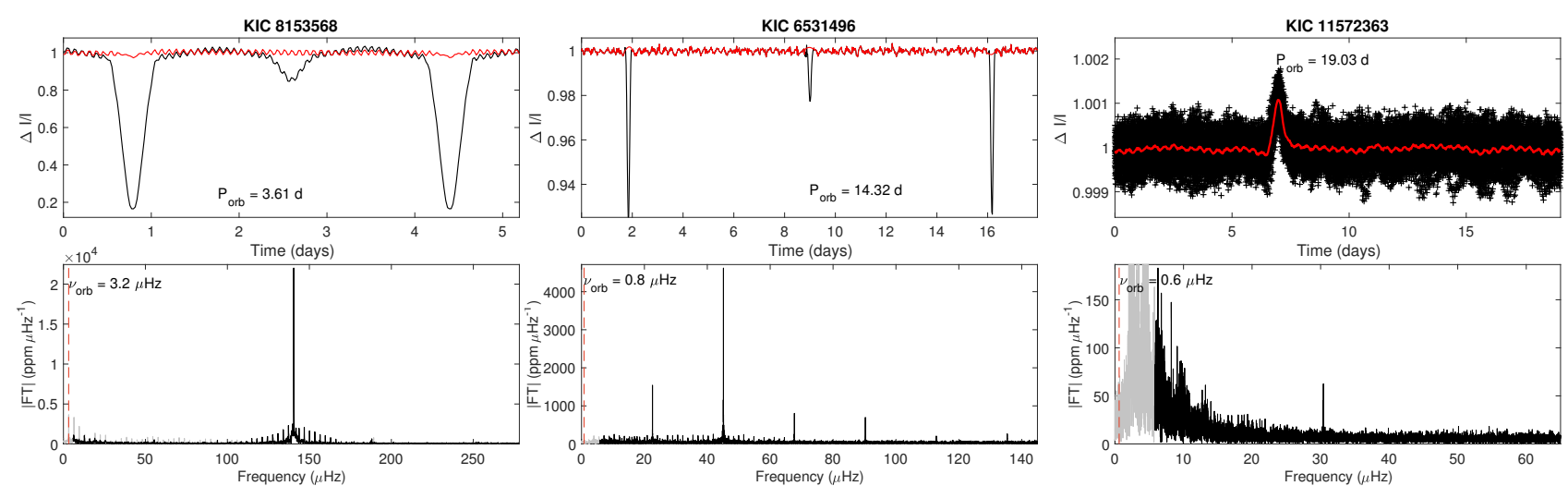

Fig. 8. Example of pulsators that either mimic tidal pulsators (KICs 8153568 and 6531496) or are actual tidal pulsators (KIC 11572363). In the cases of KIC 8153568 and 6531496 we do not plot a folded light curve but an extract of it over a little more than an orbital period (black) as the oscillations are not exactly in phase with the orbital period. The red curve is the original light curve minus the folded signal. In the case of KIC 8153568, the oscillations occur on the star that is hidden during the deeper eclipses, as the modulation is suppressed during them. Right panel: light curve and Fourier spectrum of KIC 11572363, a 19-day HB system with tidally excited oscillations.

Kepler data will be at best 19 , which is a small statistical ensemble to test a tool as widely used as asteroseismology.

To complement these systems, triple systems displaying ETVs may be a good option. Borkovits et al. (2016) showed that it is possible to estimate the mass of the $\mathrm{RG}$ and the total mass of the tight binary component of a HT system using only ETV measurements. However, the precision of such estimates is rather poor (e.g., $1.5 \pm 0.5 M_{\odot}$ for the very clear ETV KIC 7955301). To improve the precision, complementary RV measurements even in the case of SB1 systems should drastically reduce the error bars on masses - to the percent level that is required to calibrate asteroseismology. We note that no radii measurements arise from HT systems that are not triply eclipsing. The total number of HT systems for which ETVs have been detected is 13, including 4 from the present study. By considering both EBs/SB2s and HTs, the total number of systems that could be used to calibrate asteroseismic measurements of RG stars is about 30 (maximum 32) from the original Kepler mission.

Overall, to extend the sample of asteroseismic calibrators, we could use noneclipsing binaries that are both astrometric and visual binaries. Marcadon et al. (2018) studied such a system hosting a MS solar-like oscillator. In this specific case though, the $\mathrm{S} / \mathrm{N}$ of the RV measurement was not high enough to estimate the mass of the oscillating star to better than $5 \%$, which was not sufficient. Similarly to visual binaries, bright EBs could be resolved with interferometric measurements and be able to measure the proper motion of each companion, leading to the same result as for visual ones. So far, typical magnitude limitation is about eight in the visible. A few bright noneclipsing binaries may be present in the Kepler sample, and should be revealed by the ESA Gaia mission in the coming years (data release 3 or 4 planned in the early 2020s).

In addition, more data from other space missions can be considered, but none are comparable to those of Kepler. The extended Kepler mission K2 was limited to 90 days, most TESS fields of view are limited to 27 days, and CoRoT fields lasted 180 days at most. Given that Gaulme et al. (2014) showed that RG/EBs with orbits shorter than 120 days tend to show oscillation suppression, these three other options (K2, TESS, CoRoT) are less promising than Kepler, even though it will be necessary to consider them too. The ESA PLATO mission, whose launch is planned in late 2026, will surely provide new interesting targets, given its large field of view and long exposures (2 years).

\subsection{Other types of pulsators}

Beyond $\gamma$ Dor, $\delta$ Sct, and RGs we have identified 62 systems where other types of oscillations are possibly detected, most of which (59) are tidally excited. The Kepler mission has led to the discovery of a large number of tidal pulsators and has attracted much interest regarding these (e.g., Welsh et al. 2011; Dong et al. 2013; Maceroni et al. 2014; Smullen \& Kobulnicky 2015; Hambleton et al. 2013, 2016, 2018; Kjurkchieva et al. 2016; Lee et al. 2016a,b, 2017; Shporer et al. 2016; Dimitrov et al. 2017). Not surprisingly, among the 59 possible tidal pulsators that we list in Table A.1 about half (30) have already been identified as such. We note that many (35) of them are not only tidal pulsators but are also $\gamma$ Dor or $\delta$ Sct, or both. For example, KIC 4544587 was studied in detail by Hambleton et al. (2013): these authors noticed that there were self-excited pressure and gravity modes ( $\delta$ Sct types and $\gamma$ Dor respectively), but also tidally excited modes and tidally influenced $p$-modes, which were identified because their frequencies were harmonics of the orbital period. This latter study perfectly illustrates how complex the identification of tidally excited oscillations can be: several types of oscillation modes can be present and it is hard to identify and verify the tidally excited nature of some of them. In addition, harmonics of the orbital frequency can be caused by insufficient removal of the eclipse signal. This difficulty of identifying tidal oscillations was already pointed out by Aerts et al. (2010), prior to the first Kepler results.

As an example, Fig. 8 shows three examples of pulsators whose oscillation spectra resemble tidal ones, but two are not. The left panel shows an extract of the light curve and Fourier spectrum of KIC 8153568, a detached EB orbiting in 3.607 days which appears to be a typical tidal pulsator where oscillations of constant amplitude are observed all along the orbit. We note that the oscillation amplitude is suppressed during the deeper eclipses, which means that only one of the two components of the binary system oscillates. However, when folding the light curve over the orbital period, the periodic modulation averages out. The only peak that dominates the Fourier spectrum has a frequency of $v_{\text {peak }}=140.511 \mu \mathrm{Hz}$, which is not an integer multiple of the orbital frequency (factor 43.79). Tidal oscillations are expected at integer multiples of the orbital frequency because excited modes do not oscillate at their natural frequencies, but rather at tidal forcing frequencies, i.e., orbital. When a pulsation is observed at not an exact integer multiple of the orbital frequency, it is almost 

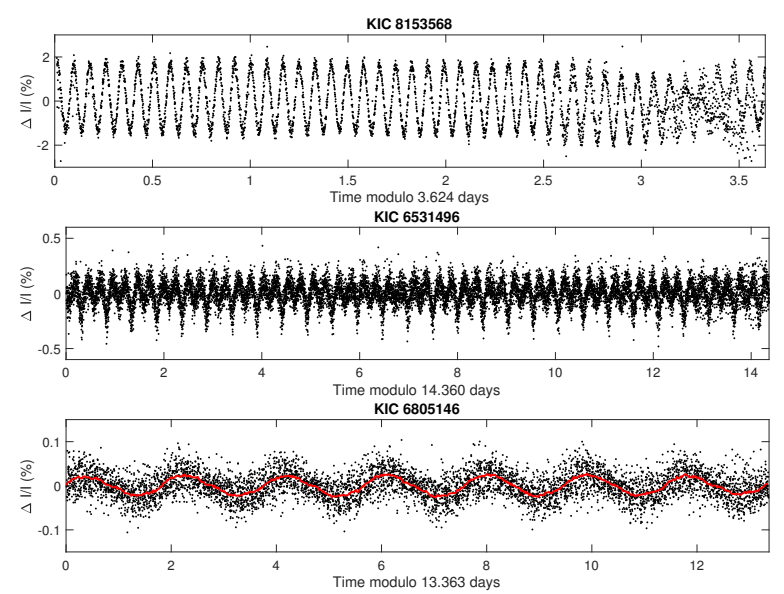

Fig. 9. Top: light curve of KIC 8153568 from Fig. 8 folded over 44 times the period corresponding to the main peak in the Fourier spectrum (3.624 days). The average eclipse signal was removed from the time series prior to folding. The region in between $t=[3,3.6]$ days corresponds with the primary eclipses. Middle: same with the light curve of KIC 6531496 from Fig. 8 folded over 28 times the period corresponding to the main peak in the Fourier spectrum (14.360 days). Bottom: light curve of KIC 6805146 folded over 7 times the period corresponding to the main peak in the Fourier spectrum (13.363 days).

certainly not a tidally excited pulsation. Also, tidally excited pulsations are almost always found in eccentric systems, which is not the case here. The effective temperature of KIC 8153568 (6800 K) makes it compatible with a $\delta$ Sct, even though the oscillation spectrum does not resemble that of a $\delta$ Sct, with only one peak surrounded by a few aliases caused by the amplitude modulation of the pulsations during the primary eclipses. Figure 9 displays the time series (where the eclipse signal is removed) folded over $44 \times 1 / v_{\text {peak }}$ (i.e., 3.624 days) and shows how the pulsation of the star is constant and coherent.

There are a handful of case similar to KIC 8153568: these also are "false" tidal oscillators, but for different reasons. For example, KIC 6531496 - a detached system with a 14.32-day orbit (Fig. 9, middle) whose light curve resembles that of KIC 8153568 - is of a different nature after further investigation. The time series displays a photometric modulation along the orbit, which appears as a series of peaks in the power spectrum. The dominant peak corresponds to a period of 0.5129 days, which is almost a twentyeighth of the orbital period. When the light curve is folded over $28 \times 0.5129$ days ( 14.36 days), a very coherent periodic signal is observed, similar to that of KIC 8153568 (Fig. 9, middle panel). However, the 0.5129-day modulation is not a sine curve but a typical OC binary light curve, which means that KIC 6531496 has no tidal oscillations but is instead either a quadruple system or two binary systems blending into each other. One could then argue that KIC 8153568 is not a binary system including a stellar pulsator, but another quadruple or another pair of blended binaries. However, the pulsation period of KIC 8153568 is 0.08 days which seems a little short to be a binary, even though the Villanova catalog presents several systems with periods down to 0.05 days. Besides, the amplitude of the photometric modulation of KIC 8153568 disappears during one of the two eclipses, which proves that it is intrinsic to one of the two stars.

Another system where the origin of a photometric modulation is unclear is the detached binary KIC 6805146 with a 13.78day orbit, which shows a sine modulation at approximately (but not exactly) $P_{\text {orb }} / 7$ (Fig. 9, bottom panel). Again, this cannot be a tidally excited mode because it is not an integer multiple of the orbital period, so the most likely option is that it is a contami- nating ellipsoidal binary with a period of 1.909 days. The final example is KIC 4677321 (described in Table A.1 but not displayed in any figure), where we observe two prominent peaks at frequencies of $(18 \pm 1 / 4) v_{\text {orb }}$, which suggest a rotational splitting where the rotation period would be $\approx 4 m P_{\text {orb }}$ where $m$ is the azimuthal order of the excited mode. Assessment of the nature of these apparently but unlikely tidal pulsations requires further study involving high-resolution spectroscopy.

Despite the series of examples of misleading cases that resemble tidally excited pulsators, most of the systems we flagged as hosting tidally excited pulsations are HB stars. The right panel of Fig. 8 displays an HB system (KIC 11572363) for which tidally excited modes are reported for the first time. There is a distinction between the HB signal and tidally excited oscillations. The $\mathrm{HB}$ signal is the result of strong gravitational distortions and heating during periastron passage and does not last the whole orbital period. Tidally excited modes are oscillations driven by the tidal force onto the internal structure of the star. It has been observed that some HB stars display tidal oscillations, which are often at exact multiples of the orbital frequency (e.g., Fig. 8, right panel), and some others do not. The latter case is often seen with HB systems including an RG star, as observed by Beck et al. (2014). We note that as observed by Thompson et al. (2012), it may be sometimes hard to determine what fraction of the peaks is due to the HB shape and what fraction is due to stellar pulsation.

Finally, a handful of systems display different types of oscillations. The KICs 5217733 and 6806632 display oscillations that resemble those of $\gamma$ Dor $/ \delta$ Sct and $\delta$ Sct, respectively, but their effective temperatures of $\approx 9200 \mathrm{~K}$ according to the KIC are a little large for this kind of pulsator (Fig. 10). They could be either $\delta$ Sct stars with overestimated temperatures or SPBs with underestimated $T_{\text {eff }}$, which we expect to be about $11000 \mathrm{~K}$. The KICs 6889235 and 8223868, also known as KOI 74 and 81 , were identified as likely white dwarfs orbiting an A and B star, respectively (Rowe et al. 2010). In both cases, oscillations are visible and are likely of tidal nature. The KIC 7749504 is an ellipsoidal binary orbiting in 0.57 days and displays clear peaks (last panel in Fig. 10) and $T_{\text {eff }} \approx 11000 \mathrm{~K}$. This system was part of the search by Balona (2015) for Maia variables that are postulated between $\delta$ Sct and SPB, and the author concluded that KIC 7749504 was a rotational variable. We confirm the presence of a series of multiple peaks at more than twice and four times the orbital period, without being able to completely discard the hypothesis of nonrotational peaks. Finally, KIC 11179657 is the only sdB pulsator observed among the EB catalog and was classified as such by Pablo et al. (2012).

\section{Conclusion}

In summary, we led the first systematic search for stellar pulsators in the likely-to-be complete Kepler EB catalog, which includes 2925 systems among which about 600 are actually ellipsoidal binaries. To this aim, we developed a dedicated data inspection tool that automatically processes the Kepler light curves, provided elementary information about EB properties is fed in (orbital period, epochs, and duration of eclipses). We focused on three main classes of stellar oscillators: classical $\delta$ Sct and $\gamma$ Dor pulsators, solar-like oscillators which happened to be all RGs, and tidal pulsators.

We first inspected the output figures produced by the DIT (e.g., Fig. 4) and made our own classification. Then, based on a manual search over the internet for possible earlier studies regarding these systems, we established a list of 303 pulsators associated with an EB, including 163 that were reported for the 

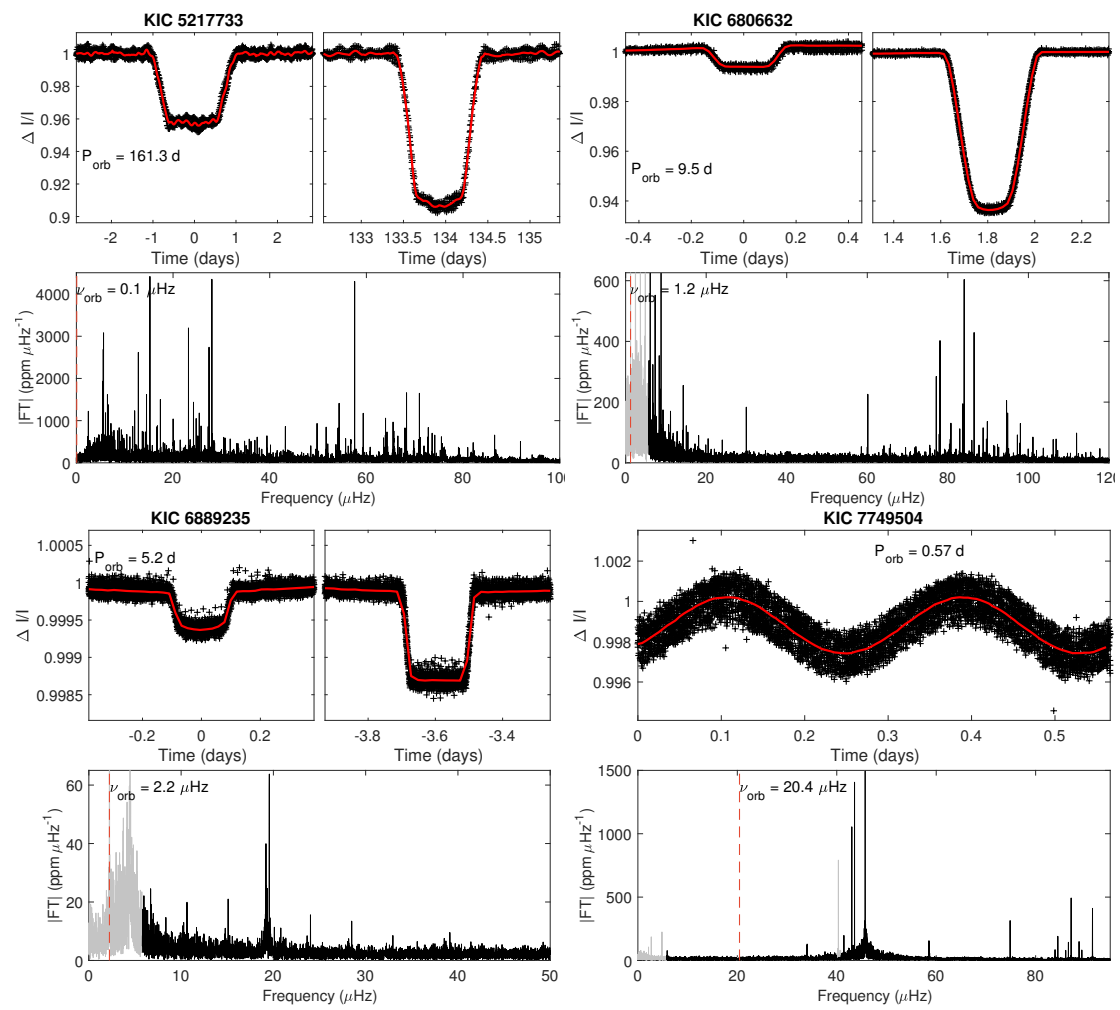

Fig. 10. Four systems in which we identify pulsations but where we have doubts about their nature (see Sect. 4.3). The KICs 5217733 and 6806632 could be either SPB or $\gamma$ Dor $\delta$ Sct. The KIC 6889235 , also known as KOI 74, is likely white dwarfs orbiting an A star with tidally excited oscillations. The KIC 7749504 is either a rotational or a Maia variable. first time as pulsator and binary candidates. A total of 149 stars are flagged as $\delta$ Sct (100 from this paper), 115 stars as $\gamma$ Dor (69 new), 85 stars as RGs (27 new), and 59 as tidally excited oscillators (29 new). There is some overlap among these groups, as some display $\delta$ Sct, $\gamma$ Dor, and tidal oscillations altogether. Many of these systems are likely to be false positives, that is, when an EB light curve is blended with a pulsator, and only deeper studies - often involving complementary RV measurements would allow us to confirm the EB nature of these candidate pulsators. False positives are expected to be more common among the shallow-eclipse and short-period systems.

Of particular interest, some very short-orbit systems $(\leq 0.5$ days) display stellar oscillations ( $\delta$ Sct and $\gamma$ Dor). Some of them are likely to be false positives, but we know from the literature that $\delta$ Sct pulsators have been identified in almost contact binaries, but not at periods of under 0.6 days. Some ground-based support will be led to determine their nature. The opportunity to detect oscillations of contact binaries would provide unique insight into these types of systems, which are indeed very common. Regarding RG oscillators, one of the current major goals is to identify a large sample of oscillators in EBs to test and calibrate asteroseismic inference on stellar masses. We were able to estimate that less than $20 \mathrm{RG} / \mathrm{EB} / \mathrm{SB} 2 \mathrm{~s}$ are present in the whole Kepler dataset, and that hierarchical triple systems may help to bring the sample up to about 30 . We also identified new tidally eccentric binaries (HBs) with tidal oscillations, as well as detached binaries that display regular coherent pulsations that resemble tidal modes, but with periods that are not integer fractions of orbital periods.

In the context of imagining the future of asteroseismology, the present discovery of stellar pulsators in EB candidates constitutes a valuable sample that merits further detailed study, especially with the help of complementary observations. Priority should be given to the brightest targets for which a fine characterization is obtained more quickly. Priority should also be given to systems that are unique, such as the three likely RG/EB/SB2s, or some short-period classical pulsators. The current authors are involved in high-resolution spectroscopic observations with the $3.5 \mathrm{~m}$ telescope of the Apache Point Observatory. However, given the large number of systems, such a survey will not be performed with only one telescope. We invite scientists that are interested to contact us in order to coordinate possible future observations.

Acknowledgements. P. Gaulme was supported in part by the German space agency (Deutsches Zentrum für Luft- und Raumfahrt) under PLATO data grant 50OO1501. P. Gaulme acknowledges NASA grant NNX17AF74G for partial support. The authors thank Jason Jackiewicz at New Mexico State University for comments and text editing. P. Gaulme thanks Cilia Damiani at Max Planck Institut für Sonnensystemforschung, Jim Fuller at Caltech, Donald W. Kurtz at University of Central Lancashire, Dominic Bowman at KU Leuven, Kosmas Gazeas at University of Athens, and Giovanni Mirouh at University of Surrey for useful comments on the manuscript. The authors thank the Apache Point Observatory 3.5-m telescope, which is owned and operated by the Astrophysical Research Consortium, for granting us observing time during quarters 2 and 3 , 2019 to support the follow up of the present work.

\section{References}

Aerts, C., Handler, G., Arentoft, T., et al. 2002, MNRAS, 333, L35 Aerts, C., Christensen-Dalsgaard, J., \& Kurtz, D. W. 2010, Asteroseismology (Berlin: Springer Science \& Business Media)

Aliçavuş, F., \& Soydugan, F. 2017, in Turkish Physical Society 32nd International Physics Congress, AIP Conf. Ser., 1815, 080003

Armstrong, D. J., Osborn, H. P., Brown, D. J. A., et al. 2014, MNRAS, 444, 1873

Baglin, A., Breger, M., Chevalier, C., et al. 1973, A\&A, 23, 221

Baglin, A., Auvergne, M., Barge, P., et al. 2009, in IAU Symposium, eds. F. Pont, D. Sasselov, \& M. J. Holman, 253, 71

Balaji, B., Croll, B., Levine, A. M., \& Rappaport, S. 2015, MNRAS, 448, 429

Balona, L. A. 2014, MNRAS, 443, 1946

Balona, L. A. 2015, MNRAS, 447, 2714

Balona, L. A., Pigulski, A., De Cat, P., et al. 2011, MNRAS, 413, 2403

Balona, L. A., Joshi, S., Joshi, Y. C., \& Sagar, R. 2013, MNRAS, 429, 1466

Balona, L. A., Daszyńska-Daszkiewicz, J., \& Pamyatnykh, A. A. 2015, MNRAS, 452, 3073

Beck, P. G., Hambleton, K., Vos, J., et al. 2014, A\&A, 564, A36

Beck, P. G., Hambleton, K., Vos, J., et al. 2015, in The Space Photometry Revolution - CoRoT Symposium 3, Kepler KASC-7 Joint Meeting, Eur. Phys. J. Web Conf., 101, 06004 
Bedding, T. R., Mosser, B., Huber, D., et al. 2011, Nature, 471, 608

Bedding, T. R., Murphy, S. J., Colman, I. L., \& Kurtz, D. W. 2015, Eur. Phys. J. Web Conf., 101, 01005

Bell, K. J., Hekker, S., \& Kuszlewicz, J. S. 2019, MNRAS, 482, 616 Biersteker, J., \& Schlichting, H. 2017, AJ, 154, 164

Bognár, Z., Lampens, P., Frémat, Y., et al. 2015, A\&A, 581, A77

Borkovits, T., Derekas, A., Fuller, J., et al. 2014, MNRAS, 443, 3068

Borkovits, T., Hajdu, T., Sztakovics, J., et al. 2016, MNRAS, 455, 4136

Borucki, W. J., Koch, D., Basri, G., et al. 2010, Science, 327, 977

Borucki, W. J., Koch, D. G., Basri, G., et al. 2011, ApJ, 736, 19

Bowman, D. M., Kurtz, D. W., Breger, M., Murphy, S. J., \& Holdsworth, D. L. 2016, MNRAS, 460, 1970

Bradley, P. A., Guzik, J. A., Miles, L. F., et al. 2015, AJ, 149, 68

Brogaard, K., Hansen, C. J., Miglio, A., et al. 2018, MNRAS, 476, 3729

Casagrande, L., Schoenrich, R., Asplund, M., et al. 2011, VizieR Online Data Catalog: $\mathrm{J} / \mathrm{A}+\mathrm{A} / 530 / \mathrm{A} 138$

Ceillier, T., Tayar, J., Mathur, S., et al. 2017, A\&A, 605, A111

Chaplin, W. J., \& Miglio, A. 2013, ARA\&A, 51, 353

Chiappini, C., Anders, F., Rodrigues, T. S., et al. 2015, A\&A, 576, L12

Conroy, K. E., Prša, A., Stassun, K. G., et al. 2014, AJ, 147, 45

Coughlin, J. L., López-Morales, M., Harrison, T. E., Ule, N., \& Hoffman, D. I 2011, AJ, 141, 78

Dal, H. A., \& Sipahi, E. 2013, PASA, 30, e016

Debosscher, J., Blomme, J., Aerts, C., \& De Ridder, J. 2011, A\&A, 529, A89

Debosscher, J., Aerts, C., Tkachenko, A., et al. 2013, A\&A, 556, A56

Demircan, O., \& Bulut, İ. 2015, in Living Together: Planets, Host Stars and Binaries, eds. S. M. Rucinski, G. Torres, \& M. Zejda, ASP Conf. Ser., 496, 395

Derekas, A., Kiss, L. L., Borkovits, T., et al. 2011, Science, 332, 216

Dimitrov, D. P., Kjurkchieva, D. P., \& Iliev, I. K. 2017, MNRAS, 469, 2089

Dodson-Robinson, S. E. 2012, ApJ, 752, 72

Dong, S., Katz, B., \& Socrates, A. 2013, ApJ, 763, L2

Faigler, S., Kull, I., Mazeh, T., et al. 2015, ApJ, 815, 26

Frandsen, S., Lehmann, H., Hekker, S., et al. 2013, A\&A, 556, A138

García Hernández, A., Moya, A., Suárez, J. C., et al. 2016, ArXiv e-prints [arXiv:1601.07374]

Gaulme, P., \& Guzik, J. A. 2014, in IAU Symposium, eds. J. A. Guzik, W. J. Chaplin, G. Handler, \& A. Pigulski, 301, 413

Gaulme, P., McKeever, J., Rawls, M. L., et al. 2013, ApJ, 767, 82

Gaulme, P., Jackiewicz, J., Appourchaux, T., \& Mosser, B. 2014, ApJ, 785, 5

Gaulme, P., McKeever, J., Jackiewicz, J., et al. 2016, ApJ, 832, 121

George, S. V., Misra, R., \& Ambika, G. 2018, Communications in Nonlinear Science and Numerical Simulation, submitted [arXiv:1805.08351]

Gies, D. R., Matson, R. A., Guo, Z., et al. 2015, AJ, 150, 178

Guo, Z., Gies, D. R., Matson, R. A., \& García Hernández, A. 2016, ApJ, 826, 69

Guo, Z., Gies, D. R., Matson, R. A., et al. 2017a, ApJ, 837, 114

Guo, Z., Gies, D. R., \& Matson, R. A. 2017b, ApJ, 851, 39

Hambleton, K. M., Kurtz, D. W., Prša, A., et al. 2013, MNRAS, 434, 925

Hambleton, K., Kurtz, D. W., Prša, A., et al. 2016, MNRAS, 463, 1199

Hambleton, K., Fuller, J., Thompson, S., et al. 2018, MNRAS, 473, 5165

Hekker, S., Debosscher, J., Huber, D., et al. 2010, ApJ, 713, L187

Hełminiak, K. G., Ukita, N., Kambe, E., \& Konacki, M. 2015, ApJ, 813, L25

Hełminiak, K. G., Ukita, N., Kambe, E., et al. 2016, MNRAS, 461, 2896

Hełminiak, K. G., Ukita, N., Kambe, E., et al. 2017a, MNRAS, 468, 1726

Hełminiak, K. G., Ukita, N., Kambe, E., et al. 2017b, A\&A, 602, A30

Kahraman Aliçavuş, F., \& Soydugan, E. 2014, in Precision Asteroseismology, eds. J. A. Guzik, W. J. Chaplin, G. Handler, \& A. Pigulski, IAU Symp., 301, 433

Kahraman Aliçavuş, F., Soydugan, E., Smalley, B., \& Kubát, J. 2017, MNRAS, 470,915

Kallinger, T., De Ridder, J., Hekker, S., et al. 2014, A\&A, 570, A41

Katsova, M. M., \& Nizamov, B. A. 2018, Geomagn. Aeron., 58, 899

Kirk, B., Conroy, K., Prša, A., et al. 2016, AJ, 151, 68

Kjeldsen, H., \& Bedding, T. R. 1995, A\&A, 293, 87

Kjurkchieva, D., \& Atanasova, T. 2016, New Astron., 49, 17

Kjurkchieva, D., Vasileva, D., \& Dimitrov, D. 2016, AJ, 152, 189

Kouzuma, S. 2018, PASJ, 70, 90

Kurtz, D. W., Shibahashi, H., Murphy, S. J., Bedding, T. R., \& Bowman, D. M. 2015a, MNRAS, 450, 3015

Kurtz, D. W., Hambleton, K. M., Shibahashi, H., Murphy, S. J., \& Prša, A. 2015b, MNRAS, 446, 1223

Kuszlewicz, J. S., North, T. S. H., Chaplin, W. J., et al. 2019, MNRAS, 487, 14

Lee, J. W., Kim, S.-L., Hong, K., Lee, C.-U., \& Koo, J.-R. 2014, AJ, 148, 37

Lee, J. W., Hong, K., \& Hinse, T. C. 2015, AJ, 149, 93

Lee, J. W., Hong, K., Kim, S.-L., \& Koo, J.-R. 2016a, MNRAS, 460, 4220

Lee, J. W., Kim, S.-L., Hong, K., et al. 2016b, AJ, 151, 25

Lee, J. W., Hong, K., Kim, S.-L., \& Koo, J.-R. 2017, ApJ, 835, 189
Lehmann, H., Southworth, J., Tkachenko, A., \& Pavlovski, K. 2013, A\&A, 557, A79

Liakos, A. 2017, A\&A, 607, A85

Liakos, A. 2018, A\&A, 616, A130

Liakos, A., \& Niarchos, P. 2017, MNRAS, 465, 1181

Lillo-Box, J., Barrado, D., Moya, A., et al. 2014, A\&A, 562, A109

Lurie, J. C., Vyhmeister, K., Hawley, S. L., et al. 2017, AJ, 154, 250

Maceroni, C., Lehmann, H., da Silva, R., et al. 2014, A\&A, 563, A59

Manuel, J., \& Hambleton, K. 2018, Am. Astron. Soc. Meet. Abstr., 231, 146.01

Marcadon, F., Appourchaux, T., \& Marques, J. P. 2018, A\&A, 617, A2

Masuda, K. 2017, AJ, 154, 64

Matijevič, G., Prša, A., Orosz, J. A., et al. 2012, AJ, 143, 123

Matson, R. A., Gies, D. R., Guo, Z., \& Williams, S. J. 2017, AJ, 154, 216

McNamara, B. J., Jackiewicz, J., \& McKeever, J. 2012, AJ, 143, 101

Mirouh, G. M., Angelou, G. C., Reese, D. R., \& Costa, G. 2019, MNRAS, 483, L28

Mosser, B., \& Appourchaux, T. 2009, A\&A, 508, 877

Moya, A., Suárez, J. C., García Hernández, A., \& Mendoza, M. A. 2017, MNRAS, 471, 2491

Murphy, S. J., Bedding, T. R., Niemczura, E., Kurtz, D. W., \& Smalley, B. 2015, MNRAS, 447, 3948

Murphy, S. J., Moe, M., Kurtz, D. W., et al. 2018, MNRAS, 474, 4322

Niemczura, E., Murphy, S. J., Smalley, B., et al. 2015, MNRAS, 450, 2764

Niemczura, E., Polińska, M., Murphy, S. J., et al. 2017, MNRAS, 470, 2870

Orosz, J. A. 2015, in Living Together: Planets, Host Stars and Binaries, eds. S. M. Rucinski, G. Torres, \& M. Zejda, ASP Conf. Ser., 496, 55

Özdarcan, O., \& Dal, H. A. 2017, PASA, 34, e017

Pablo, H., Kawaler, S. D., Reed, M. D., et al. 2012, MNRAS, 422, 1343

Pinsonneault, M. H., An, D., Molenda-Żakowicz, J., et al. 2012, ApJS, 199, 30

Prša, A. 2018, Modeling and Analysis of Eclipsing Binary Stars; The Theory and Design Principles of PHOEBE (Bristol: IOP Publishing)

Prša, A., Guinan, E. F., Devinney, E. J., et al. 2008, ApJ, 687, 542

Prša, A., Batalha, N., Slawson, R. W., et al. 2011, AJ, 141, 83

Ramsay, G., Brooks, A., Hakala, P., et al. 2014, MNRAS, 437, 132

Rappaport, S., Deck, K., Levine, A., et al. 2013, ApJ, 768, 33

Rappaport, S., Nelson, L., Levine, A., et al. 2015, ApJ, 803, 82

Rawls, M. L., Gaulme, P., McKeever, J., et al. 2016, ApJ, 818, 108

Ricker, G. R., Winn, J. N., Vanderspek, R., et al. 2015, J. Astron. Telesc. Instrum. Syst., 1, 014003

Rowe, J. F., Borucki, W. J., Koch, D., et al. 2010, ApJ, 713, L150

Rowe, J. F., Coughlin, J. L., Antoci, V., et al. 2015, ApJS, 217, 16

Saio, H., Kurtz, D. W., Murphy, S. J., Antoci, V. L., \& Lee, U. 2018, MNRAS, 474, 2774

Sandquist, E. L., Jessen-Hansen, J., Shetrone, M. D., et al. 2016, ApJ, 831, 11

Santerne, A., Moutou, C., Tsantaki, M., et al. 2016, A\&A, 587, A64

Shibahashi, H., \& Kurtz, D. W. 2012, MNRAS, 422, 738

Shporer, A., Fuller, J., Isaacson, H., et al. 2016, ApJ, 829, 34

Sipahi, E., \& Dal, H. A. 2014, New Astron., 26, 62

Slawson, R. W., Prša, A., Welsh, W. F., et al. 2011, AJ, 142, 160

Smullen, R. A., \& Kobulnicky, H. A. 2015, ApJ, 808, 166

Southworth, J. 2013, A\&A, 557, A119

Southworth, J. 2015, Eur. Phys. J. Web Conf., 101, 04001

Southworth, J., Zima, W., Aerts, C., et al. 2011, MNRAS, 414, 2413

Sowicka, P., Handler, G., Debski, B., et al. 2017, MNRAS, 467, 4663

Soydugan, E., Soydugan, F., Demircan, O., \& İbanoğlu, C. 2006, MNRAS, 370, 2013

Szatmary, K. 1990, J. Am. Assoc. Var. Star Obs. (JAAVSO), 19, 52

Themeß1, N., Hekker, S., Southworth, J., et al. 2018, MNRAS, 478, 4669

Thompson, S. E., Everett, M., Mullally, F., et al. 2012, ApJ, 753, 86

Turner, G. 2019, New Astron., 67, 40

Turner, G., \& Holaday, J. 2015, J. Am. Assoc. Var. Star Obs. (JAAVSO), 43, 40 Turner, G., \& Maynard, A. 2016, ArXiv e-prints [arXiv:1604 . 04867]

Twicken, J. D., Chandrasekaran, H., Jenkins, J. M., et al. 2010, in Society of Photo-Optical Instrumentation Engineers (SPIE) Conference Series, Proc. SPIE, 7740, 77401U

Uytterhoeven, K., Moya, A., Grigahcène, A., et al. 2011, A\&A, 534, A125

Van Reeth, T., Tkachenko, A., Aerts, C., et al. 2015a, A\&A, 574, A17

Van Reeth, T., Tkachenko, A., Aerts, C., et al. 2015b, ApJS, 218, 27

Welsh, W. F., Orosz, J. A., Aerts, C., et al. 2011, ApJS, 197, 4

Yakut, K. 2015, IAU Gen. Assem., 22, 2256344

Zhang, X. B., Luo, Y. P., Wang, K., \& Luo, C. Q. 2015, AJ, 150, 37

Zhang, J., Qian, S.-B., \& He, J.-D. 2017a, Res. Astron. Astrophys., 17, 22

Zhang, J., Qian, S.-B., Wang, S.-M., Wu, Y., \& Jiang, L.-Q. 2017b, PASJ, 69, 49

Zhang, X. B., Fu, J. N., Liu, N., Luo, C. Q., \& Ren, A. B. 2017c, ApJ, 850, 125 Zhou, A. Y. 2010, ArXiv e-prints [arXiv:1002 .2729]

Zimmerman, M. K., Thompson, S. E., Mullally, F., et al. 2017, ApJ, 846, 147 


\section{Appendix A: Table of pulsators in EBs}

Table A.1. Properties of the systems where pulsations are detected.

\begin{tabular}{|c|c|c|c|c|c|c|c|c|c|c|c|c|c|}
\hline KIC & $\begin{array}{l}T_{\text {eff }} \\
{[\mathrm{K}]}\end{array}$ & $K_{\text {mag }}$ & Type & Ecl & $\begin{array}{l}P_{\text {orb }} \\
\text { [day] }\end{array}$ & $\begin{array}{l}\text { Depth } \\
{[\%]}\end{array}$ & Sep & $\frac{W_{2}}{W_{1}}$ & $\begin{array}{l}\frac{W_{1}+W_{2}}{P_{\text {orb }}} \\
{[\%]}\end{array}$ & Puls type & Literature & New PB & Notes \\
\hline 1295531 & $\ldots$ & $\ldots$ & ELL & 0 & 1.69 & 0.97 & $\ldots$ & $\ldots$ & $\ldots$ & $\gamma$ Dor & & $\mathrm{Y}$ & \\
\hline 2162283 & 6683 & 9.55 & ELL & 0 & 0.91 & 1.07 & $\ldots$ & $\ldots$ & $\ldots$ & $\gamma$ Dor, $\delta$ Sct & Nie17 & & \\
\hline 2306740 & 5647 & 13.54 & $\mathrm{D}$ & 2 & 10.31 & 31.53 & 0.52 & 0.54 & 6.0 & Rotation(?), Tidal(?) & Yak15 & & \\
\hline 2444348 & 4546 & 10.32 & $\mathrm{D}$ & 0 & 103.21 & 0.07 & $\ldots$ & $\ldots$ & $\ldots$ & RG & Bck14 & & $\mathrm{HB}$ \\
\hline 2697935 & 4883 & 10.63 & $\mathrm{D}$ & 1 & 21.51 & 0.24 & $\ldots$ & $\ldots$ & $\ldots$ & RG & Bck14 & & $\mathrm{HB}$ \\
\hline 2711114 & 6281 & 12.34 & $\mathrm{D}$ & 2 & 2.86 & 0.33 & 0.50 & 0.93 & 6.4 & RG & & $\mathrm{Y}$ & FP or triple \\
\hline 2720096 & 4832 & 13.05 & $\mathrm{D}$ & 0 & 26.67 & 0.00 & $\ldots$ & $\ldots$ & $\ldots$ & RG & Bck14 & & $\mathrm{HB}$ \\
\hline 2720354 & 6513 & 13.12 & $\mathrm{D}$ & 2 & 2.82 & 7.35 & 0.50 & 0.57 & 10.8 & $\gamma$ Dor, Rossby(?) & & & Villanova Pu \\
\hline 2970804 & $\ldots$ & 9.16 & SD & 2 & 0.69 & 0.71 & $\ldots$ & $\ldots$ & $\ldots$ & $\gamma$ Dor, $\delta$ Sct, Rossby(?) & & $\mathrm{Y}$ & \\
\hline 2997455 & 4795 & 13.80 & $\mathrm{D}$ & 2 & 1.13 & 0.45 & $\ldots$ & $\ldots$ & $\ldots$ & RG & Gau13 & & FP or triple \\
\hline 2998124 & 6374 & 13.12 & $\mathrm{D}$ & 2 & 28.60 & 8.94 & 0.47 & 1.21 & 2.4 & $\delta$ Sct & & $\mathrm{Y}$ & \\
\hline 3228863 & 6561 & 11.82 & SD & 2 & 0.73 & 48.21 & 0.50 & 0.76 & 27.9 & $\gamma$ Dor & Lee14 & & \\
\hline 3327980 & 7321 & 12.12 & $\mathrm{D}$ & 2 & 4.23 & 42.54 & 0.50 & 1.04 & 11.5 & $\delta$ Sct, Rossby(?) & Ali17 & $\mathrm{Y}$ & \\
\hline 3441784 & $\ldots$ & 9.73 & $\mathrm{D}$ & 1 & 52.57 & 2.44 & $\ldots$ & $\ldots$ & $\ldots$ & $\delta$ Sct & & $\mathrm{Y}$ & KOI 976 \\
\hline 3547874 & 6371 & 10.95 & $\mathrm{D}$ & 0 & 19.69 & $\ldots$ & $\ldots$ & $\ldots$ & $\ldots$ & Tidal & Tho12, Zim17 & & $\mathrm{HB}, P_{\mathrm{orb}}$ harm., spots \\
\hline 3556229 & $\ldots$ & 17.66 & SD & 2 & 0.80 & 15.45 & 0.50 & 0.87 & 27.8 & Tidal? & & $\mathrm{Y}$ & \\
\hline 3735597 & 6556 & 12.24 & SD & 2 & 1.97 & 18.77 & 0.50 & 0.90 & 25.7 & $\gamma$ Dor, $\delta$ Sct & & $\mathrm{Y}$ & \\
\hline 3749404 & 7144 & 10.57 & $\mathrm{D}$ & 0 & 20.31 & $\ldots$ & $\ldots$ & $\ldots$ & $\ldots$ & Tidal & Ham16 & & $\mathrm{HB}, P_{\text {orb }}$ harm., triple \\
\hline 3761175 & 6890 & 12.83 & ELL & 0 & 5.16 & 0.18 & $\ldots$ & $\ldots$ & $\ldots$ & RG or $\gamma$ Dor & & $\mathrm{Y}$ & $\gamma$ Dor $T_{\text {eff }}$, RG osc. \\
\hline 3851949 & 4981 & 13.94 & $\mathrm{D}$ & 2 & 54.77 & 0.73 & 0.64 & 0.70 & 5.7 & RG & & $\mathrm{Y}$ & $T_{2}<T_{1}$, depleted $\ell=1$, \\
\hline 3858884 & $\ldots$ & 9.28 & $\mathrm{D}$ & 2 & 25.95 & 37.31 & 0.77 & 1.42 & 5.0 & $\delta$ Sct, Tidal & Mac14 & & \\
\hline 3863594 & $\ldots$ & $\ldots$ & $\mathrm{OC}$ & 0 & 0.05 & 0.00 & $\ldots$ & $\ldots$ & $\ldots$ & $\gamma$ Dor $/ \delta$ Sct & & $\mathrm{Y}$ & Likely FP (shallow ecl) \\
\hline 3867593 & 7037 & 13.56 & $\mathrm{D}$ & 1 & 73.34 & 9.46 & $\ldots$ & $\ldots$ & $\ldots$ & $\gamma$ Dor & Deb11 & & \\
\hline 3869326 & 4903 & 11.60 & $\mathrm{D}$ & 2 & 8.59 & 0.08 & $\ldots$ & $\ldots$ & $\ldots$ & RG & Bed11 & $\mathrm{Y}$ & Blend or triple (shallow ecl) \\
\hline 3869825 & 6476 & 13.32 & $\mathrm{D}$ & 2 & 4.80 & 1.56 & 0.47 & 2.43 & 25.4 & $\gamma$ Dor, $\delta$ Sct & & $\mathrm{Y}$ & \\
\hline 4054905 & 4702 & 12.98 & $\mathrm{D}$ & 2 & 274.71 & 16.83 & 0.31 & 0.61 & 2.9 & RG & Ben+ & & SB2 \\
\hline 4055765 & 6440 & 12.60 & $\mathrm{D}$ & 1 & 9.97 & 0.15 & $\ldots$ & $\ldots$ & $\ldots$ & $\gamma$ Dor & KN18 & $\mathrm{Y}$ & KOI 100 , flares, $P_{\text {orb }}=P_{\text {orb }} / 2$ \\
\hline 4078157 & 5547 & 15.48 & $\mathrm{D}$ & 2 & 16.02 & 1.22 & $\ldots$ & $\ldots$ & $\ldots$ & RG & Brk16 & $\mathrm{Y}$ & Triple (ETVs), sec. ecl. \\
\hline
\end{tabular}

Notes. Systems are sorted by increasing KIC number (first column). Second and third columns are the effective temperatures, and Kepler magnitudes from the KIC. The "Type" column indicates the type of binary systems: D for detached, SD for semi-detached, OC for over-contact, and ELL for ellipsoidal. The "Ecl" column indicates the number of eclipses that are visible per orbital period (0 for ELL systems, 1 or 2 for the others). Column 6 displays the orbital period $P_{\text {orb }}$ expressed in days rounded at two digits. Columns 7-10 indicate the eclipse depth of the deepest eclipse (primary) expressed in percent, the orbital phase separation "sep" in between the secondary and the primary eclipse, the eclipse duration ratio $W_{2} / W_{1}$, and the sum of eclipse duration relative to orbital period $\left(W_{2}+W_{1}\right) / P_{\text {orb }}$. The pulsator type "Puls type" is either $\delta$-Sct for $\delta$ Scuti, $\gamma$ Dor for $\gamma$ Doradus, "Rossby", "RG" for RG solar-like oscillator, "tidal", SPB for slowly pulsating B-type stars, "WD" for white dwarf, or sdB for subdwarf B. The type "rot" stands for surface stellar "rotation" that may mimic stellar pulsations. The literature references are compacted (see below). "New PB" indicates a target identified both as a pulsator and a binary. Systems identified for the first time are marked with a "Y". Notes are random relevant specific information regarding each system. "HB" stands for heartbeat, FP for false positive, KOI for Kepler object of interest, $P_{\text {orb }}$ for orbital period, "Ecl." for eclipse, "harm." for harmonics, $T_{1}$ and $T_{2}$ for star 1 star 2 effective temperatures, $T_{\text {eff }}$ refers to the published KIC effective temperature, "SB1" and "SB2" for single or double-lined spectroscopic binary. The label " $P_{\text {orb }}=P_{\text {orb }} / 2$ " means that the actual orbital period is half of what Villanova database reported.

References. AS14: Kahraman Aliçavuş et al. (2014), Ali17: Aliçavuş \& Soydugan (2017), Arm14: Armstrong et al. (2014), Blj15: Balaji et al. (2015), Bal11: Balona et al. (2011), Bal13: Balona et al. (2013), Bal14: Balona (2014), Bal15: Balona (2015), Bal15b: Balona et al. (2015), Bck14: Beck et al. (2014), Bed11: Bedding et al. (2011), Bel19: Bell et al. (2019), Bog15: Bognár et al. (2015), Bow16: Bowman et al. (2016), Bie17: Biersteker \& Schlichting (2017), Bor11: Borucki et al. (2011), Brk14: Borkovits et al. (2014), Brk16: Borkovits et al. (2016), Bra15: Bradley et al. (2015), Bro18: Brogaard et al. (2018), Cei17: Ceillier et al. (2017), Con14: Conroy et al. (2014), Cou11: Coughlin et al. (2011), Deb11: Debosscher et al. (2011), Deb13: Debosscher et al. (2013), Dem15: Demircan \& Bulut (2015), Dim17: Dimitrov et al. (2017), DR12: Dodson-Robinson (2012), Don13: Dong et al. (2013), Fra13: Frandsen et al. (2013), Gau13: Gaulme et al. (2013), Gau14: Gaulme et al. (2014), Gau16: Gaulme et al. (2016), GG14: Gaulme \& Guzik (2014), Gie15: Gies et al. (2015), Guo16: Guo et al. (2016), Guo17: Guo et al. (2017a), Guo17b: Guo et al. (2017b), Fai15: Faigler et al. (2015), Geo18: George et al. (2018), Hek10: Hekker et al. (2010), Ham13: Hambleton et al. (2013), Ham16: Hambleton et al. (2016), Ham18: Hambleton et al. (2018), Hel16: Hełminiak et al. (2016), Hel17: Hełminiak et al. (2017b), Hel17b: Hełminiak et al. (2017a), KN18: Katsova \& Nizamov (2018), Kju16: Kjurkchieva et al. (2016), Kju16b: Kjurkchieva \& Atanasova (2016), Kou18: Kouzuma (2018), Kur15: Kurtz et al. (2015b), Kus19: Kuszlewicz et al. (2019) Lee14: Lee et al. (2014), Lee15: Lee et al. (2015), Lee16a: Lee et al. (2016a), Lee16b: Lee et al. (2016b), Lee17: Lee et al. (2017), Leh13: Lehmann et al. (2013), LiB14: Lillo-Box et al. (2014), Lur17: Lurie et al. (2017), Lia17: Liakos (2017), Lia18: Liakos (2018), LN17: Liakos \& Niarchos (2017), Mac14: Maceroni et al. (2014), MH18: Manuel \& Hambleton (2018), Mas17: Masuda (2017), Mat17: Matson et al. (2017), McN12: McNamara et al. (2012), Moy17: Moya et al. (2017), Mur15: Murphy et al. (2015), Mur18: Murphy et al. (2018), Nie15: Niemczura et al. (2015), Nie17: Niemczura et al. (2017), Ozd17: Özdarcan \& Dal (2017), Oro15: Orosz (2015), Pab12: Pablo et al. (2012), Ram14: Ramsay et al. (2014), Rap13: Rappaport et al. (2013), Rap15: Rappaport et al. (2015), Raw16: Rawls et al. (2016), Row10: Rowe et al. (2010), Row15: Rowe et al. (2015), Sow17: Sowicka et al. (2017), Sai18: Saio et al. (2018), Snd16: Sandquist et al. (2016), San16: Santerne et al. (2016), Shp16: Shporer et al. (2016), Smu15: Smullen \& Kobulnicky (2015), Sou11: Southworth et al. (2011), Sou15: Southworth (2015), The18: Themeß1 et al. (2018), Tho12: Thompson et al. (2012), TM16: Turner \& Maynard (2016), Tur15: Turner \& Holaday (2015), Tur19: Turner (2019), Uyt11: Uytterhoeven et al. (2011), Wel11: Welsh et al. (2011), Yak15: Yakut (2015), Zha17: Zhang et al. (2017a), Zha17b: Zhang et al. (2017b), ZXB17: Zhang et al. (2017c), Zho10: Zhou (2010), Zim17: Zimmerman et al. (2017) 
Table A.1. continued.

\begin{tabular}{|c|c|c|c|c|c|c|c|c|c|c|c|c|c|}
\hline KIC & $\begin{array}{l}T_{\text {eff }} \\
{[\mathrm{K}]}\end{array}$ & $K_{\text {mag }}$ & Type & $\mathrm{Ecl}$ & $\begin{array}{l}P_{\text {orb }} \\
{[\text { day }]}\end{array}$ & $\begin{array}{l}\text { Depth } \\
{[\%]}\end{array}$ & Sep & $\frac{W_{2}}{W_{1}}$ & $\begin{array}{l}\frac{W_{1}+W_{2}}{P_{\text {orb }}} \\
{[\%]}\end{array}$ & Puls type & Literature & New $P B$ & Notes \\
\hline 4142768 & 5401 & 12.12 & $\mathrm{D}$ & 1 & 27.99 & 1.75 & $\ldots$ & $\ldots$ & $\ldots$ & $\gamma$ Dor, $\delta$ Sct, Tidal & MH18 & & $T_{\text {eff }}$ cold, HB \\
\hline 4142768 & 5401 & 12.12 & $\mathrm{D}$ & 1 & 27.99 & 1.75 & $\ldots$ & $\ldots$ & $\ldots$ & $\gamma$ Dor, $\delta$ Sct, Tidal & MH18 & & $\mathrm{HB}, P_{\text {orb }}$ harm., $T_{\text {eff }}$ cool \\
\hline 4150611 & 6623 & 7.90 & $\mathrm{D}$ & 3 & $\begin{array}{l}1.52 \\
8.65 \\
94.20\end{array}$ & 0.36 & $\ldots$ & $\ldots$ & $\begin{array}{l}\ldots \\
\ldots \\
\ldots\end{array}$ & $\gamma$ Dor, $\delta$ Sct & Hel17 & & Quintuple \\
\hline 4245897 & 6595 & 12.54 & SD & 2 & 11.26 & 75.69 & 0.50 & 0.54 & 11.9 & Tidal?, $\delta$ Sct? & & $\mathrm{Y}$ & \\
\hline 4253860 & 6636 & 12.65 & $\mathrm{D}$ & 0 & 155.06 & 0.22 & $\ldots$ & $\ldots$ & $\ldots$ & $\gamma$ Dor, $\delta$ Sct & & $\mathrm{Y}$ & HB \\
\hline 4360072 & 4950 & 11.16 & $\mathrm{D}$ & 2 & 1086.50 & 0.61 & 0.44 & 0.90 & 1.0 & $\mathrm{RG}$ & Ben+ & & SB1 \\
\hline 4544587 & 8255 & 10.80 & $\mathrm{D}$ & 2 & 2.19 & 44.79 & 0.65 & 0.75 & 15.1 & $\gamma$ Dor, $\delta$ Sct, Tidal & Ham13 & & \\
\hline 4570326 & $\ldots$ & 9.77 & ELL & 0 & 1.12 & 4.73 & $\ldots$ & $\ldots$ & $\ldots$ & $\delta \mathrm{Sct}$ & GG14 & & \\
\hline 4659476 & 6129 & 13.22 & $\mathrm{D}$ & 0 & 59.00 & $\ldots$ & $\ldots$ & $\ldots$ & $\ldots$ & $\gamma$ Dor, Tidal & Guo $\mathrm{PhD}$ & & HB \\
\hline 4663623 & 4735 & 12.83 & $\mathrm{D}$ & 2 & 358.10 & 12.49 & 0.50 & 0.96 & 1.5 & RG & Gau14, Gau16 & & SB2 \\
\hline 4677321 & 5979 & 13.48 & SD & 2 & 1.57 & 9.94 & 0.50 & 0.92 & 24.7 & Tidal & & $\mathrm{Y}$ & Two prominent peaks at $(18 \pm 1 / 4) v_{\text {orb }}$ \\
\hline 4739791 & 7538 & 14.70 & SD & 2 & 0.90 & 16.14 & 0.50 & 0.95 & 23.9 & $\delta$ Sct, Tidal & Lee16b & & $P_{\text {orb }}$ harm., R CMa-type EB \\
\hline 4758368 & 4594 & 10.80 & $\mathrm{D}$ & 2 & 3.75 & 3.62 & 0.50 & 0.96 & 17.7 & $\mathrm{RG}, \delta$ Sct, Tidal & Gau13, Zha17b & & Triple (ETVs) \\
\hline 4769799 & 4911 & 10.95 & $\mathrm{D}$ & 2 & 21.93 & 2.22 & $\ldots$ & $\ldots$ & $\ldots$ & RG & Brk16 & $\mathrm{Y}$ & Triple (ETVs), sec. ecl. \\
\hline 4851217 & 6694 & 11.11 & SD & 2 & 2.47 & 19.80 & 0.48 & 0.97 & 19.4 & $\delta$ Sct & Mat17 & $\mathrm{Y}$ & \\
\hline 4932691 & 7109 & 13.63 & $\mathrm{D}$ & 2 & 18.11 & 11.14 & 0.26 & 0.80 & 2.4 & $\gamma$ Dor, Tidal & Kju16 & & \\
\hline 4947528 & 6828 & 13.91 & $\mathrm{OC}$ & 22 & 0.50 & 17.60 & $\ldots$ & $\ldots$ & $\ldots$ & $\gamma$ Dor, Rossby(?) & & $\mathrm{Y}$ & \\
\hline 4949187 & 6307 & 13.82 & $\mathrm{D}$ & 0 & 11.98 & 0.08 & $\ldots$ & $\ldots$ & $\ldots$ & Tidal & Zim17 & & $\mathrm{HB}, P_{\text {orb }}$ harm. \\
\hline 4949194 & 8444 & 8.94 & $\mathrm{D}$ & 0 & 41.26 & $\ldots$ & $\ldots$ & $\ldots$ & $\ldots$ & Tidal & Don 13 & & $\mathrm{HB}, P_{\text {orb }}$ harm. \\
\hline 4949770 & 7907 & 12.57 & ELL & 2 & 1.56 & 13.54 & 0.50 & $\ldots$ & $\ldots$ & $\gamma$ Dor, $\delta$ Sct, Rossby(?) & & $\mathrm{Y}$ & $T_{\text {eff }}$ too hot \\
\hline 4954113 & 7630 & 11.93 & ELL & 0 & 0.67 & 3.31 & $\ldots$ & $\ldots$ & $\ldots$ & $\delta$ Sct & & $\mathrm{Y}$ & \\
\hline 4999260 & 5048 & 9.33 & $\mathrm{OC}$ & 22 & 0.38 & 2.35 & $\ldots$ & $\ldots$ & $\ldots$ & RG & Zho10, Gau13, SchSt+ & & Triple \\
\hline 5006817 & 4935 & 10.87 & $\mathrm{D}$ & 0 & 94.81 & 0.18 & $\ldots$ & $\ldots$ & $\ldots$ & RG & Bck14 & & $\mathrm{HB}$ \\
\hline 5024450 & 5945 & 15.06 & $\mathrm{D}$ & 2 & 3.05 & 2.23 & 0.50 & 1.06 & 7.6 & $\delta$ Sct & Bal13 & & FP from nearby $\delta$ Sct \\
\hline 5034333 & 8444 & 11.52 & $\mathrm{D}$ & 0 & 6.93 & 0.43 & $\ldots$ & $\ldots$ & $\ldots$ & Tidal & Tho12, Zim17 & & $\mathrm{HB}$ \\
\hline 5039392 & 4152 & 11.65 & $\mathrm{D}$ & 0 & 236.73 & 1.44 & $\ldots$ & $\ldots$ & $\ldots$ & RG & Bck14 & & HB \\
\hline 5179609 & 4777 & 12.78 & $\mathrm{D}$ & 2 & 43.93 & 0.97 & $\ldots$ & $\ldots$ & $\ldots$ & RG & Gau13, Gau16 & & SB1 \\
\hline 5197256 & 7609 & 11.02 & OC & 0 & 6.96 & 1.04 & $\ldots$ & $\ldots$ & $\ldots$ & $\delta$ Sct & Tur15 & & \\
\hline 5198315 & $\ldots$ & $\ldots$ & $\mathrm{OC}$ & 0 & 3.64 & 3.36 & $\ldots$ & $\ldots$ & $\ldots$ & $\gamma$ Dor? & & $\mathrm{Y}$ & Bump at $[18,28] \mu \mathrm{Hz}$ \\
\hline 5211385 & 8010 & 12.68 & ELL & 0 & 1.66 & 6.58 & $\ldots$ & $\ldots$ & $\ldots$ & $\gamma$ Dor, $\delta$ Sct & & $\mathrm{Y}$ & $T_{\text {eff }}$ hot \\
\hline 5211470 & 4525 & 13.09 & $\mathrm{D}$ & 2 & 4.81 & 0.91 & 0.50 & 0.85 & 6.2 & RG & & $\mathrm{Y}$ & Likely FP (no ETVs) \\
\hline 5217733 & 9116 & 7.39 & $\mathrm{D}$ & 2 & 161.25 & 10.09 & 0.17 & 2.03 & 1.9 & SPB or Tidal & & $\mathrm{Y}$ & Hot, flat bottom ecl + high $e$ \\
\hline 5218014 & 4752 & 12.92 & $\mathrm{D}$ & 2 & 10.85 & 0.68 & 0.36 & 1.23 & 3.3 & RG, Tidal(?) & Zho10, Gau13 & & Triple? FP? \\
\hline 5296276 & 5711 & 13.79 & ELL & 0 & 1.89 & 3.47 & $\ldots$ & $\ldots$ & $\ldots$ & Tidal & & $\mathrm{Y}$ & \\
\hline 5302006 & 6536 & 15.05 & ELL & 0 & 0.15 & 0.23 & $\ldots$ & $\ldots$ & $\ldots$ & $\delta$ Sct & Bal14 & & Triple \\
\hline 5308778 & 4812 & 11.78 & $\mathrm{D}$ & 2 & 40.57 & 0.21 & 0.50 & 1.05 & 7.8 & RG & Gau13, Gau16 & & SB1 \\
\hline 5310435 & 6215 & 13.25 & $\mathrm{SD}$ & 2 & 4.93 & 26.57 & 0.50 & 0.64 & 13.4 & $\delta$ Sct & & $\mathrm{Y}$ & \\
\hline 5371109 & 7396 & 13.97 & ELL & 0 & 1.19 & 5.15 & $\ldots$ & $\ldots$ & $\ldots$ & $\delta$ Sct & & $\mathrm{Y}$ & \\
\hline 5384713 & 3690 & 13.69 & $\mathrm{D}$ & 1 & 60.33 & 0.27 & $\ldots$ & $\ldots$ & $\ldots$ & Tidal & & $\mathrm{Y}$ & $P_{\text {orb }}$ harm., spots, flares \\
\hline 5467126 & 4683 & 12.37 & $\mathrm{D}$ & 2 & 2.85 & 0.00 & 0.50 & 1.00 & 25.1 & RG & Cou11 & $\mathrm{Y}$ & Likely FP \\
\hline 5475712 & 6370 & 13.28 & $\mathrm{SD}$ & 2 & 2.99 & 13.53 & 0.50 & 0.96 & 17.0 & $\delta$ Sct? & & $\mathrm{Y}$ & \\
\hline 5479973 & 6169 & 14.89 & SD & 2 & 1.80 & 14.25 & 0.50 & 0.80 & 19.7 & $\delta$ Sct & & $\mathrm{Y}$ & $T_{\text {eff }}$ cold \\
\hline 5560831 & $\ldots$ & 16.09 & SD & 2 & 0.87 & 11.84 & 0.50 & 0.67 & 19.0 & $\gamma$ Dor, $\delta$ Sct, or Tidal & & $\mathrm{Y}$ & \\
\hline 5560831 & $\ldots$ & 16.09 & SD & 2 & 0.87 & 11.84 & 0.50 & 0.67 & 19.0 & $\gamma$ Dor $/ \delta$ Sct?, Tidal? & & $\mathrm{Y}$ & Triple (ETVs) \\
\hline 5565486 & 6471 & 14.96 & $\mathrm{D}$ & 2 & 2.83 & 17.41 & 0.50 & 1.09 & 13.8 & $\gamma$ Dor, $\delta$ Sct & Lur17, Zha17 & & \\
\hline 5621294 & 8425 & 13.61 & SD & 2 & 0.94 & 36.47 & 0.50 & 0.93 & 28.8 & $\delta$ Sct & Lee15 & $\mathrm{Y}$ & $T_{\text {eff hot }}$ \\
\hline 5623923 & 8300 & 16.52 & SD & 2 & 1.21 & 8.23 & 0.50 & 0.76 & 19.8 & $\delta$ Sct & Ram14 & & $T_{\text {eff }}$ hot \\
\hline 5640750 & 4557 & 11.56 & $\mathrm{D}$ & 2 & 987.30 & 7.41 & 0.67 & 1.29 & 1.8 & $\mathrm{RG}$ & Gau13, The18 & & SB2 \\
\hline 5733154 & 6839 & 12.67 & $\mathrm{D}$ & 0 & 62.52 & $\ldots$ & $\ldots$ & $\ldots$ & $\ldots$ & $\delta$ Sct, Tidal & & $\mathrm{Y}$ & $\mathrm{HB}$ \\
\hline 5736537 & 7395 & 13.65 & $\mathrm{D}$ & 0 & 1.76 & 1.09 & $\ldots$ & $\ldots$ & $\ldots$ & $\delta$ Sct & & $\mathrm{Y}$ & Peaks are rather evenly spaced \\
\hline 5786154 & 4743 & 13.53 & $\mathrm{D}$ & 2 & 197.92 & 7.83 & 0.28 & 0.77 & 4.7 & RG & Gau13, Gau16 & & SB2 \\
\hline 5790807 & 6349 & 9.95 & $\mathrm{D}$ & 1 & 80.00 & 2.60 & $\ldots$ & $\ldots$ & $\ldots$ & $\gamma$ Dor & Zim17 & $\mathrm{Y}$ & $\mathrm{HB}$, rotation \\
\hline 5809827 & 6076 & 13.54 & SD & 2 & 1.22 & 16.10 & 0.50 & 1.07 & 23.4 & $\gamma$ Dor, Rossby(?) & & $\mathrm{Y}$ & \\
\hline 5812701 & 6419 & 11.35 & $\mathrm{D}$ & 1 & 17.86 & 1.05 & $\ldots$ & $\ldots$ & $\ldots$ & $\gamma$ Dor & Mas17, Bie17 & $\mathrm{Y}$ & Kepler- $448 \mathrm{~b}, P_{\text {orb }}=P_{\text {orb }} / 2$ \\
\hline 5817566 & 7994 & 11.68 & $\mathrm{D}$ & 2 & 4.21 & 15.58 & 0.51 & 0.91 & 16.9 & $\delta$ Sct & & $\mathrm{Y}$ & $P_{\text {orb }}=P_{\text {orb }} / 2$, sec. ecl. \\
\hline 5866138 & 5078 & 11.07 & $\mathrm{D}$ & 2 & 342.30 & 0.43 & $\ldots$ & $\ldots$ & $\ldots$ & RG & Ben+ & & SB1 \\
\hline 5872506 & 7571 & 14.73 & $\mathrm{OC}$ & 0 & 2.13 & 3.87 & $\ldots$ & $\ldots$ & $\ldots$ & $\delta$ Sct & & $\mathrm{Y}$ & \\
\hline 5872696 & $\ldots$ & 11.85 & ELL & 0 & 0.17 & 0.62 & $\ldots$ & $\ldots$ & $\ldots$ & $\delta$ Sct & & $\mathrm{Y}$ & Very short $P_{\text {orb }}$ \\
\hline 5877364 & $\ldots$ & 8.88 & $\mathrm{D}$ & 0 & 89.65 & $\ldots$ & $\ldots$ & $\ldots$ & $\ldots$ & RG & Shp16 & $\mathrm{Y}$ & $\mathrm{HB}$ \\
\hline 5944240 & 7474 & 14.67 & $\mathrm{D}$ & 0 & 2.55 & 1.61 & $\ldots$ & $\ldots$ & $\ldots$ & $\delta$ Sct & & $\mathrm{Y}$ & No ecl (ell?) \\
\hline 5952403 & $\ldots$ & 6.97 & SD & 2 & 0.91 & 0.32 & $\ldots$ & $\ldots$ & $\ldots$ & Tidal & Sou 15 & & Triply ecl. syst. \\
\hline 5960989 & 6074 & 12.51 & $\mathrm{D}$ & 0 & 50.72 & $\ldots$ & $\ldots$ & $\ldots$ & $\ldots$ & Tidal & Shp16 & & $\mathrm{HB}, P_{\text {orb }}$ harm. \\
\hline 5962514 & 6566 & 14.84 & SD & 2 & 1.58 & 21.44 & 0.50 & 0.95 & 19.4 & $\gamma$ Dor & & $\mathrm{Y}$ & \\
\hline 5977736 & 7661 & 13.17 & $\mathrm{OC}$ & 0 & 1.58 & 2.13 & $\ldots$ & $\ldots$ & $\ldots$ & $\delta$ Sct & & $\mathrm{Y}$ & No ecl (ell?) \\
\hline 5983348 & 5680 & 15.05 & $\mathrm{D}$ & 2 & 25.15 & 3.61 & 0.50 & 0.78 & 2.0 & Tidal? & & $\mathrm{Y}$ & Sparse spectrum \\
\hline 5990753 & 4886 & 10.92 & $\mathrm{D}$ & 2 & 7.22 & 0.00 & $\ldots$ & $\ldots$ & $\ldots$ & RG & RG/EB Cei17 & & Likely FP (shallow ecl) \\
\hline 5991936 & 8630 & 13.42 & ELL & 0 & 0.81 & 0.10 & $\ldots$ & $\ldots$ & $\ldots$ & $\delta$ Sct? & Bal14 & & Likely FP (shallow ecl), $T_{\text {eff }}$ hot \\
\hline 6042191 & 4986 & 13.16 & $\mathrm{D}$ & 1 & 43.39 & $\ldots$ & $\ldots$ & $\ldots$ & $\ldots$ & RG & & $\mathrm{Y}$ & $\mathrm{HB}, \ell=1$ depleted \\
\hline 6063448 & 6416 & 13.76 & $\mathrm{D}$ & 2 & 76.02 & 7.78 & 0.78 & 0.81 & 1.1 & $\delta$ Sct, $\gamma$ Dor(?) & Lur17 & & \\
\hline
\end{tabular}


Table A.1. continued.

\begin{tabular}{|c|c|c|c|c|c|c|c|c|c|c|c|c|c|}
\hline KIC & $\begin{array}{l}T_{\text {eff }} \\
{[\mathrm{K}]}\end{array}$ & $K_{\text {mag }}$ & Type & Ecl & $\begin{array}{l}P_{\text {orb }} \\
{[\text { day }]}\end{array}$ & $\begin{array}{l}\text { Depth } \\
{[\%]}\end{array}$ & Sep & $\frac{W_{2}}{W_{1}}$ & $\begin{array}{l}\frac{W_{1}+W_{2}}{P_{\text {orb }}} \\
{[\%]}\end{array}$ & Puls type & Literature & New PB & Notes \\
\hline 6109688 & 6845 & 12.27 & $\mathrm{D}$ & 2 & 14.09 & 10.73 & 0.55 & 0.52 & 4.1 & $\gamma$ Dor, $\delta$ Sct & Lur17 & & \\
\hline 6117415 & 6282 & 10.54 & $\mathrm{D}$ & 1 & 19.74 & 8.47 & $\ldots$ & $\ldots$ & $\ldots$ & Tidal? & $\begin{array}{l}\text { Zhao Guo, } \\
\text { Valentina Schmid PhDs }\end{array}$ & & \\
\hline 6145939 & 6090 & 12.37 & $\mathrm{D}$ & 1 & 17.75 & 7.98 & $\ldots$ & $\ldots$ & $\ldots$ & $\gamma$ Dor, $\delta$ Sct, Tidal & Lur17 & & HB \\
\hline 6220497 & 7254 & 14.75 & SD & 2 & 1.32 & 37.02 & 0.50 & 0.92 & 31.4 & $\delta$ Sct, Tidal & Lee16a & & \\
\hline 6262882 & 8317 & 13.98 & $\mathrm{OC}$ & 0 & 1.00 & 1.94 & $\ldots$ & $\ldots$ & $\ldots$ & $\delta$ Sct or Tidal & & $\mathrm{Y}$ & $T_{\text {eff }}$ hot for $\delta$ Sct, not OC but ELL \\
\hline 6286155 & 5062 & 13.76 & $\mathrm{D}$ & 2 & 14.54 & 0.41 & 0.46 & 1.78 & 3.9 & RG & & $\mathrm{Y}$ & Likely FP, $\ell=1$ depleted \\
\hline 6292398 & $\ldots$ & $\ldots$ & $\mathrm{D}$ & 0 & 9.24 & 0.04 & $\ldots$ & $\ldots$ & $\ldots$ & $\gamma$ Dor, $\delta \operatorname{Sct}(?)$ & Mur15 & $\mathrm{Y}$ & $\mathrm{HB}$ \\
\hline 6302592 & 7737 & 13.85 & ELL & 2 & 1.58 & 3.67 & 0.49 & $\ldots$ & $\ldots$ & $\delta$ Sct & Zho10 & & \\
\hline 6311681 & 5500 & 15.36 & SD & 2 & 0.84 & 0.60 & $\ldots$ & $\ldots$ & $\ldots$ & RG?, $\gamma$ Dor? & & $\mathrm{Y}$ & Likely FP (shallow ecl) \\
\hline 6390205 & 8254 & 12.54 & $\mathrm{OC}$ & 0 & 0.45 & 5.64 & $\ldots$ & $\ldots$ & $\ldots$ & $\delta \mathrm{Sct}$ & & $\mathrm{Y}$ & Very short orbit: triple? \\
\hline 6525209 & 5207 & 14.66 & $\mathrm{D}$ & 2 & 75.13 & 12.40 & 0.22 & 0.51 & 1.6 & RG & Row15 & $\mathrm{Y}$ & $T_{2}<T_{1}$, low SNR osc. \\
\hline 6531496 & 5604 & 16.09 & $\mathrm{D}$ & 2 & 14.32 & 5.82 & 0.50 & 1.19 & 2.9 & Tidal(?), Quadruple(?) & & $\mathrm{Y}$ & \\
\hline 6549491 & 5597 & 14.91 & $\mathrm{OC}$ & 0 & 0.76 & 0.95 & $\ldots$ & $\ldots$ & $\ldots$ & Rotation(?), $\delta \operatorname{Sct}(?)$ & Blj15 & $\mathrm{Y}$ & $T_{\text {eff }}$ cool \\
\hline 6613627 & 7090 & 12.55 & ELL & 0 & 0.15 & 0.67 & $\ldots$ & $\ldots$ & $\ldots$ & $\gamma$ Dor $/ \delta$ Sct & Bow16 & & Very short $P_{\text {orb }}$ : FP, triple? \\
\hline 6629588 & 6520 & 13.98 & SD & 2 & 2.26 & 20.10 & 0.50 & 0.94 & 18.3 & $\delta$ Sct & LN17 & & \\
\hline 6669809 & 7239 & 10.76 & SD & 2 & 0.73 & 24.24 & 0.50 & 0.72 & 26.3 & $\delta$ Sct & Brk16 & $\mathrm{Y}$ & Triple (ETVs) \\
\hline 6757558 & 4742 & 12.87 & $\mathrm{D}$ & 1 & 421.16 & 0.35 & $\ldots$ & $\ldots$ & $\ldots$ & RG & Ben+ & & SB1 \\
\hline 6762188 & 4801 & 13.67 & $\mathrm{D}$ & 1 & 7.16 & 0.27 & $\ldots$ & $\ldots$ & $\ldots$ & $\mathrm{RG}$ & Zho10, Gau13 & & Triple (ETVs) \\
\hline 6775034 & 6886 & 13.99 & $\mathrm{D}$ & 0 & 10.03 & $\ldots$ & $\ldots$ & $\ldots$ & $\ldots$ & $\gamma \operatorname{Dor}(?)$, Tidal(?) & Shp16, Zim17 & & $\mathrm{HB}$, spots \\
\hline 6805146 & 6214 & 13.21 & $\mathrm{D}$ & 2 & 13.78 & 2.82 & $\ldots$ & $\ldots$ & $\ldots$ & Tidal(?), rotation(?) & & $\mathrm{Y}$ & Sec. ecl., flat bottom ecl. \\
\hline 6806632 & 9224 & 13.29 & $\mathrm{D}$ & 2 & 9.47 & 6.92 & 0.70 & 0.90 & 7.2 & $\mathrm{SPB}(?), \delta \mathrm{Sct}(?)$ & & $\mathrm{Y}$ & $\begin{array}{l}\text { Hot for } \delta \text { Sct, cold for SPB, } \\
\text { flat bottom ecl, HB }\end{array}$ \\
\hline 6847018 & 6211 & 13.34 & $\mathrm{D}$ & 2 & 16.66 & 2.75 & $\ldots$ & $\ldots$ & $\ldots$ & Tidal & & $\mathrm{Y}$ & HB, $P_{\text {orb }}$ harm., sec. ecl. \\
\hline 6850665 & 4828 & 12.39 & $\mathrm{D}$ & 1 & 214.72 & 0.66 & $\ldots$ & $\ldots$ & $\ldots$ & RG & & $\mathrm{Y}$ & HB with ecl. \\
\hline 6852488 & 7262 & 13.91 & SD & 2 & 2.17 & 17.72 & 0.51 & 0.90 & 18.0 & $\delta$ Sct & & $\mathrm{Y}$ & \\
\hline 6889235 & 9288 & 10.96 & $\mathrm{D}$ & 2 & 5.19 & 0.16 & 0.50 & 1.14 & 9.9 & WD? SPB? & Row10 & $\mathrm{Y}$ & KOI 74 \\
\hline 7023917 & $\ldots$ & 10.12 & $\mathrm{OC}$ & 2 & 0.77 & 7.35 & 0.50 & $\ldots$ & $\ldots$ & $\delta$ Sct & & $\mathrm{Y}$ & Short orbit \\
\hline 7037405 & 4605 & 11.88 & $\mathrm{D}$ & 2 & 207.15 & 6.86 & 0.40 & 1.16 & 5.1 & RG & Gau13, Gau16 & & SB2 \\
\hline 7060333 & 7576 & 9.09 & ELL & 0 & 2.53 & 0.36 & $\ldots$ & $\ldots$ & $\ldots$ & $\gamma$ Dor, $\delta$ Sct & Nie15 & & \\
\hline 7220322 & 4887 & 11.88 & $\mathrm{D}$ & 2 & 0.75 & 0.00 & 0.50 & 1.00 & 20.9 & Tidal & & $\mathrm{Y}$ & $P_{\text {orb harm., spots }}$ \\
\hline 7368103 & 7838 & 13.42 & SD & 2 & 2.18 & 12.25 & 0.50 & 0.83 & 16.9 & $\gamma$ Dor, $\delta$ Sct & Zhao Guo PhD & & $T_{\text {eff }}$ little hot \\
\hline 7377422 & 4488 & 13.56 & $\mathrm{D}$ & 2 & 107.62 & 1.90 & 0.23 & 0.94 & 4.1 & RG & Gau13, Gau16 & & SB2, low SNR osc. \\
\hline 7385478 & 6477 & 11.47 & SD & 2 & 1.66 & 19.01 & 0.50 & 0.83 & 16.0 & $\gamma$ Dor & Ozd17 & & \\
\hline 7422883 & 6639 & 11.25 & $\mathrm{D}$ & 2 & 11.41 & 4.76 & $\ldots$ & $\ldots$ & $\ldots$ & $\gamma$ Dor & Deb11 & & Sec. ecl. \\
\hline 7431665 & 4729 & 10.97 & $\mathrm{D}$ & 1 & 281.40 & 0.58 & $\ldots$ & $\ldots$ & $\ldots$ & RG & Bck14 & & HB with ecl. \\
\hline 7515679 & 7127 & 12.25 & ELL & 1 & 5.55 & 0.00 & $\ldots$ & $\cdots$ & $\ldots$ & $\gamma$ Dor & & & Kepler-1517b, Villanova $\mathrm{Pu}$ \\
\hline 7622486 & $\cdots$ & 13.09 & SD & 3 & $\begin{array}{l}2.28 \\
40.25\end{array}$ & 1.92 & $\cdots$ & $\cdots$ & $\ldots$ & $\delta \operatorname{Sct}(?)$ & Zha17 & $\mathrm{Y}$ & Triple triply eclipsing \\
\hline 7676610 & 8493 & 12.64 & ELL & 0 & 1.23 & 3.73 & $\ldots$ & $\ldots$ & $\ldots$ & $\delta$ Sct & Blj15 & $\mathrm{Y}$ & $T_{\text {eff }}$ hot, short orbit \\
\hline 7700578 & 6693 & 14.15 & SD & 2 & 1.51 & 47.80 & 0.50 & 0.96 & 22.2 & $\delta$ Sct & Bra 15 & $\mathrm{Y}$ & \\
\hline 7749504 & 11064 & 12.72 & ELL & 0 & 0.57 & 0.27 & $\ldots$ & $\ldots$ & $\ldots$ & Maia var(?), Rot(?) & McN12, Bal15 & $\mathrm{Y}$ & B-type star (ref) \\
\hline 7765894 & 7233 & 11.96 & ELL & 0 & 3.20 & 4.16 & $\ldots$ & $\ldots$ & $\ldots$ & $\delta$ Sct & & $\mathrm{Y}$ & \\
\hline 7767774 & $\ldots$ & 12.51 & ELL & 0 & 0.17 & 0.33 & $\ldots$ & $\ldots$ & $\cdots$ & $\gamma$ Dor, $\delta$ Sct & & $\mathrm{Y}$ & Very short $P_{\text {orb}}:$ FP, triple? \\
\hline 7768447 & 4694 & 11.90 & $\mathrm{D}$ & 2 & 122.50 & 0.64 & 0.31 & 1.21 & 4.2 & RG & & $\mathrm{Y}$ & Likely FP (shallow ecl) \\
\hline 7769072 & 4858 & 13.89 & $\mathrm{D}$ & 2 & 0.61 & 0.19 & 0.50 & 0.98 & 16.8 & RG & Cou11 & $\mathrm{Y}$ & Likely FP (shallow ecl) \\
\hline 7799540 & 5177 & 12.39 & $\mathrm{D}$ & 0 & 60.00 & 0.00 & $\ldots$ & $\ldots$ & $\ldots$ & RG & Bck14 & & $\mathrm{HB}$ \\
\hline 7833144 & 7724 & 12.56 & $\mathrm{D}$ & 0 & 2.25 & 3.32 & $\ldots$ & $\ldots$ & $\ldots$ & $\gamma$ Dor(?), $\delta$ Sct, Tidal & & $\mathrm{Y}$ & Broad excess power at $30-50 \mu \mathrm{Hz}$ \\
\hline 7881722 & 7762 & 13.59 & $\mathrm{D}$ & 0 & 0.95 & 1.47 & $\ldots$ & $\ldots$ & $\ldots$ & $\delta$ Sct & & $\mathrm{Y}$ & no ecl. (ell) \\
\hline 7914906 & 6952 & 11.96 & $\mathrm{D}$ & 1 & 8.75 & 4.08 & $\ldots$ & $\ldots$ & $\ldots$ & $\gamma$ Dor, $\delta$ Sct, Tidal & Bal14 & $\mathrm{Y}$ & HB \\
\hline 7955301 & 4821 & 12.67 & $\mathrm{D}$ & 2 & 15.32 & 1.18 & 0.49 & 1.34 & 6.4 & RG & Cou11, Gau13, Rap13, Brk16 & & Triple (ETVs) \\
\hline 7976783 & 7937 & 11.99 & ELL & 0 & 1.21 & 0.57 & $\ldots$ & $\ldots$ & $\ldots$ & $\gamma$ Dor, $\delta$ Sct & & $\mathrm{Y}$ & \\
\hline 8045121 & 6990 & 11.97 & ELL & 0 & 0.26 & 0.53 & $\ldots$ & $\ldots$ & $\ldots$ & $\gamma$ Dor, $\delta$ Sct & Conroy $\mathrm{PhD}$, Brk16 & & Triple \\
\hline 8054233 & 4733 & 11.78 & $\mathrm{D}$ & 2 & 1058.00 & 3.81 & 0.41 & 1.29 & 1.2 & RG & Gau14, Gau16 & & SB1(?) \\
\hline 8087799 & 7869 & 14.17 & $\mathrm{OC}$ & 2 & 0.93 & 5.07 & 0.50 & $\ldots$ & $\ldots$ & $\mathrm{WD}+\delta \mathrm{Sct}$ & ZXB17 & & \\
\hline 8095275 & 4683 & 13.61 & $\mathrm{D}$ & 0 & 23.01 & 0.34 & $\ldots$ & $\ldots$ & $\ldots$ & $\mathrm{RG}$ & Gau13, Bck14 & & $\mathrm{HB}$ \\
\hline 8112039 & $\ldots$ & $\ldots$ & $\mathrm{D}$ & 0 & 41.81 & $\ldots$ & $\ldots$ & $\ldots$ & $\ldots$ & Tidal & Wel11 & & $P_{\text {orb }}$ harm., historic HB KOI-54 \\
\hline 8113154 & 6589 & 12.90 & SD & 2 & 2.59 & 3.08 & $\ldots$ & $\ldots$ & $\ldots$ & $\gamma$ Dor, $\delta$ Sct & Arm14 & $\mathrm{Y}$ & Circumbinary planets \\
\hline 8129189 & 5080 & 12.48 & $\mathrm{D}$ & 2 & 53.65 & 21.57 & 0.63 & 0.97 & 2.7 & RG & & $\mathrm{Y}$ & small RG+RG/SG? \\
\hline 8143170 & 4957 & 12.85 & $\mathrm{D}$ & 2 & 28.79 & 5.09 & 0.46 & 1.13 & 4.2 & RG & Brk16, Bal15 & $\mathrm{Y}$ & Triple (ETVs), flares \\
\hline 8144355 & 4880 & 13.68 & $\mathrm{D}$ & 0 & 80.51 & $\ldots$ & $\ldots$ & $\ldots$ & $\ldots$ & RG & Bck14 & & $\mathrm{HB}$ \\
\hline 8153568 & 6803 & 15.08 & SD & 2 & 3.61 & 82.49 & 0.50 & 0.84 & 21.1 & Tidal & Bra15 & $\mathrm{Y}$ & \\
\hline 8164262 & 7487 & 13.36 & $\mathrm{D}$ & 0 & 87.46 & 0.19 & $\ldots$ & $\ldots$ & $\ldots$ & Tidal g-modes & Ham18, Sai18 & & $\mathrm{HB}, P_{\text {orb }}$ harm. \\
\hline 8182360 & 6904 & 15.32 & SD & 2 & 0.70 & 9.22 & 0.50 & 0.96 & 36.7 & $\delta$ Sct & Bra15 & $\mathrm{Y}$ & \\
\hline 8193315 & 6457 & 13.70 & $\mathrm{D}$ & 2 & 2.62 & 22.40 & 0.50 & 1.01 & 12.4 & $\gamma$ Dor, $\delta \mathrm{Sct}$ & & $\mathrm{Y}$ & \\
\hline 8197761 & 7068 & 10.66 & $\mathrm{D}$ & 1 & 19.74 & 0.49 & $\ldots$ & $\ldots$ & $\ldots$ & $\gamma$ Dor, $\delta$ Sct & Sow17 & & \\
\hline 8210370 & 4793 & 11.17 & $\mathrm{D}$ & 0 & 153.70 & 0.64 & $\ldots$ & $\ldots$ & $\ldots$ & RG & Bck14 & & $\mathrm{HB}$ \\
\hline 8219268 & 4712 & 12.49 & SD & 1 & 6.25 & 0.05 & $\ldots$ & $\ldots$ & $\ldots$ & RG & LiB14 & $\mathrm{Y}$ & Kepler-91b,c (no EB) \\
\hline 8240109 & 7740 & 13.49 & SD & 2 & 2.30 & 11.29 & 0.50 & 1.78 & 18.3 & $\gamma \operatorname{Dor}(?), \delta$ Sct & & $\mathrm{Y}$ & \\
\hline 8262223 & 7596 & 12.15 & SD & 2 & 1.61 & 15.58 & 0.50 & 1.04 & 16.6 & $\delta$ Sct & Guo17 & & pre-WD $+\delta$ Sct \\
\hline 8264510 & 7478 & 10.61 & $\mathrm{D}$ & 0 & 5.69 & $\ldots$ & $\ldots$ & $\ldots$ & $\ldots$ & $\delta \operatorname{Sct}(?)$, Tidal & Tho12 & $\mathrm{Y}$ & HB \\
\hline
\end{tabular}


Table A.1. continued.

\begin{tabular}{|c|c|c|c|c|c|c|c|c|c|c|c|c|c|}
\hline KIC & $\begin{array}{l}T_{\text {eff }} \\
{[\mathrm{K}]}\end{array}$ & $K_{\mathrm{mag}}$ & Type & Ecl & $\begin{array}{l}P_{\text {orb }} \\
\text { [day] }\end{array}$ & $\begin{array}{l}\text { Depth } \\
{[\%]}\end{array}$ & Sep & $\frac{W_{2}}{W_{1}}$ & $\begin{array}{l}\frac{W_{1}+W_{2}}{P_{\text {orb }}} \\
{[\%]}\end{array}$ & Puls type & Literature & New PB & Notes \\
\hline 8308347 & 4826 & 15.47 & $\mathrm{D}$ & 2 & 164.95 & 6.41 & 0.61 & 0.50 & 3.2 & $\mathrm{RG}$ & & $\mathrm{Y}$ & $T_{2}>T_{1}$ \\
\hline 8330092 & 6902 & 13.48 & OC & 22 & 0.32 & 3.71 & $\ldots$ & $\ldots$ & $\ldots$ & $\gamma$ Dor, $\delta$ Sct & Bal13 & $\mathrm{Y}$ & OC, short orbit \\
\hline 8410637 & 4682 & 10.77 & $\mathrm{D}$ & 2 & 408.32 & 10.47 & 0.23 & 3.48 & 2.8 & RG & Hek10, Fra13 & & SB2 \\
\hline 8429450 & $\ldots$ & 13.11 & $\mathrm{D}$ & 2 & 2.71 & 34.95 & 0.50 & 1.00 & 12.8 & $\gamma$ Dor, $\delta$ Sct & Oro15 & $\mathrm{Y}$ & Triple (ETVs) \\
\hline 8430105 & 4965 & 10.42 & $\mathrm{D}$ & 2 & 63.33 & 1.97 & 0.34 & 0.98 & 6.7 & $\mathrm{RG}$ & Gau14, Gau16 & & SB2 \\
\hline 8430210 & 8636 & 12.45 & ELL & 0 & 1.98 & 0.58 & $\ldots$ & $\ldots$ & $\ldots$ & $\gamma$ Dor, $\delta$ Sct, or Tidal & & $\mathrm{Y}$ & $T_{\text {eff }}$ hot \\
\hline 8452840 & 6473 & 12.59 & ELL & 0 & 1.20 & 0.28 & $\ldots$ & $\ldots$ & $\ldots$ & $\gamma$ Dor & Gau13 & & \\
\hline 8453324 & 4733 & 11.52 & $\mathrm{D}$ & 0 & 2.52 & 0.74 & $\ldots$ & $\ldots$ & $\ldots$ & $\mathrm{RG}$ & Zho10, Gau13 & & Likely FP \\
\hline 8454250 & $\ldots$ & 12.78 & $\mathrm{D}$ & 1 & 5.08 & 0.23 & $\cdots$ & $\cdots$ & $\cdots$ & $\gamma$ Dor, $\delta$ Sct & & $\mathrm{Y}$ & Exoplanet? \\
\hline 8455359 & 6645 & 14.21 & OC & 0 & 2.96 & 2.52 & $\ldots$ & $\ldots$ & $\ldots$ & $\gamma$ Dor, $\delta$ Sct & & $\mathrm{Y}$ & \\
\hline 8456774 & 6757 & 13.22 & $\mathrm{D}$ & 0 & 2.89 & $\ldots$ & $\ldots$ & $\ldots$ & $\ldots$ & $\delta$ Sct, Tidal & Zhao Guo PhD & $\mathrm{Y}$ & HB \\
\hline 8459354 & 7433 & 11.14 & $\mathrm{D}$ & 0 & 53.56 & 0.24 & $\ldots$ & $\ldots$ & $\ldots$ & $\gamma$ Dor, $\delta$ Sct, Tidal & Moy 17 & & $\mathrm{HB}$ \\
\hline 8504570 & 6874 & 13.25 & $\mathrm{D}$ & 2 & 4.01 & 16.56 & 0.50 & 1.11 & 10.0 & $\gamma$ Dor, $\delta$ Sct & & $\mathrm{Y}$ & \\
\hline 8548416 & $\ldots$ & 13.34 & ELL & 0 & 1.16 & 3.01 & $\ldots$ & $\ldots$ & $\ldots$ & $\gamma$ Dor & & $\mathrm{Y}$ & \\
\hline 8553788 & 8045 & 12.69 & $\mathrm{SD}$ & 2 & 1.61 & 14.66 & 0.50 & 0.90 & 18.5 & $\delta \mathrm{Sct}$ & Lia18 & & $T_{\text {eff }}$ hot, Algol type \\
\hline 8560861 & 7647 & 8.50 & $\mathrm{D}$ & 2 & 31.97 & 7.12 & 0.52 & 1.03 & 3.0 & $\gamma$ Dor & Brk14 & & \\
\hline 8561192 & 6852 & 16.27 & $\mathrm{SD}$ & 2 & 2.74 & 65.76 & 0.50 & 0.93 & 21.7 & $\delta$ Sct or Tidal & & $\mathrm{Y}$ & Strong $180-\mu \mathrm{Hz}$ signal \\
\hline 8563964 & $\ldots$ & 12.94 & ELL & 0 & 0.34 & 1.11 & $\ldots$ & $\ldots$ & $\ldots$ & $\gamma$ Dor & Brk16 & $\mathrm{Y}$ & Triple (ETVs) \\
\hline 8564976 & 4726 & 13.23 & $\mathrm{D}$ & 1 & 152.83 & 0.22 & $\ldots$ & $\ldots$ & $\ldots$ & RG & Kus 19 & & HB, KOI 3890 \\
\hline 8569819 & 7137 & 13.04 & $\mathrm{D}$ & 2 & 20.85 & 31.31 & 0.50 & 0.42 & 3.3 & $\gamma \operatorname{Dor}(?), \delta$ Sct & Kur15 & & \\
\hline 8685306 & 6971 & 11.79 & ELL & 0 & 0.81 & 3.02 & $\ldots$ & $\ldots$ & $\ldots$ & $\gamma$ Dor, $\delta$ Sct? & Kou18 & $\mathrm{Y}$ & $\mathrm{OC}$ \\
\hline 8702921 & 4824 & 11.98 & $\mathrm{D}$ & 2 & 19.38 & 0.52 & 0.44 & 4.38 & 26.0 & $\mathrm{RG}$ & Gau14, Gau16 & & SB1 \\
\hline 8703887 & $\ldots$ & 11.05 & $\mathrm{D}$ & 1 & 14.17 & 1.54 & $\ldots$ & $\ldots$ & $\ldots$ & $\delta$ Sct $\gamma$ Dor, Tidal & & $\mathrm{Y}$ & $\mathrm{HB}$ \\
\hline 8719324 & $\ldots$ & 11.61 & $\mathrm{D}$ & 0 & 10.23 & $\ldots$ & $\ldots$ & $\ldots$ & $\ldots$ & Tidal & Tho12 & & $\mathrm{HB}, P_{\text {orb }}$ harm. \\
\hline 8719419 & 6642 & 12.93 & $\mathrm{D}$ & 1 & 12.63 & 3.56 & $\ldots$ & $\ldots$ & $\ldots$ & $\gamma$ Dor & & $\mathrm{Y}$ & Unconfirmed plan cand. \\
\hline 8800998 & 8616 & 13.72 & ELL & 0 & 0.88 & 1.71 & $\ldots$ & $\ldots$ & $\ldots$ & $\delta$ Sct & Geo18 & $\mathrm{Y}$ & $T_{\text {eff }}$ hot \\
\hline 8803882 & 5018 & 13.00 & $\mathrm{D}$ & 0 & 89.63 & 0.00 & $\ldots$ & $\ldots$ & $\ldots$ & RG & Bck14 & & $\mathrm{HB}$ \\
\hline 8823868 & 9751 & 11.41 & $\mathrm{D}$ & 2 & 23.88 & 0.58 & 0.50 & 0.75 & 3.6 & Tidal(?), SPB(?) & Row10 & & KOI 81, WD+B \\
\hline 8848288 & 4624 & 9.84 & $\mathrm{SD}$ & 1 & 5.57 & 0.08 & $\ldots$ & $\ldots$ & $\ldots$ & RG & Zho10, Gau13 & & Likely FP, exoplanet? \\
\hline 8894630 & 7117 & 11.54 & ELL & 1 & 1.08 & 3.97 & $\ldots$ & $\ldots$ & $\ldots$ & $\delta$ Sct & & $\mathrm{Y}$ & \\
\hline 8912308 & 4872 & 11.43 & $\mathrm{D}$ & 0 & 20.17 & $\ldots$ & $\cdots$ & $\cdots$ & $\cdots$ & RG & Bck14 & & $\mathrm{HB}$ \\
\hline 8912468 & 6194 & 11.75 & ELL & 0 & 0.09 & 0.41 & $\ldots$ & $\ldots$ & $\ldots$ & $\gamma$ Dor, $\delta$ Sct & & $\mathrm{Y}$ & $T_{\text {eff }}$ cool, Very short $P_{\text {orb }}$ \\
\hline 9016693 & 7020 & 11.63 & $\mathrm{D}$ & 0 & 26.37 & $\ldots$ & $\ldots$ & $\ldots$ & $\ldots$ & Tidal & Shp16 & & $\mathrm{HB}, P_{\text {orb }}$ harm. \\
\hline 9101279 & 8372 & 13.95 & $\mathrm{SD}$ & 2 & 1.81 & 90.09 & 0.50 & 0.82 & 23.9 & $\delta$ Sct & & $\mathrm{Y}$ & $T_{\text {eff }}$ hot \\
\hline 9108058 & 6563 & 14.28 & SD & 2 & 2.17 & 61.88 & 0.50 & 0.78 & 21.5 & $\delta$ Sct & Bra15 & $\mathrm{Y}$ & \\
\hline 9108579 & 6386 & 11.56 & ELL & 0 & 1.17 & 2.02 & $\ldots$ & $\ldots$ & $\ldots$ & $\gamma$ Dor & & $\mathrm{Y}$ & \\
\hline 9151763 & 4307 & 11.70 & $\mathrm{D}$ & 0 & 438.05 & $\ldots$ & $\ldots$ & $\ldots$ & $\ldots$ & RG & Bck14 & & НB \\
\hline 9153621 & 4789 & 12.87 & $\mathrm{D}$ & 2 & 305.82 & 3.19 & 0.16 & 3.04 & 3.3 & RG & Ben+ & & SB2 \\
\hline 9159301 & 7959 & 12.15 & SD & 2 & 3.04 & 48.88 & 0.50 & 0.89 & 20.7 & $\delta$ Sct & Guo PhD, Mat17 & & SB2 \\
\hline 9163796 & 5135 & 9.60 & $\mathrm{D}$ & 0 & 121.01 & $\ldots$ & $\ldots$ & $\ldots$ & $\ldots$ & $\mathrm{RG}$ & Bck14 & & $\mathrm{HB}$ \\
\hline 9164561 & 8059 & 13.71 & SD & 2 & 1.27 & 4.82 & 0.50 & 0.97 & 20.7 & $\gamma$ Dor, $\delta$ Sct? & Rap15 & $\mathrm{Y}$ & $\mathrm{WD}, T_{\mathrm{eff}}$ hot \\
\hline 9181877 & 4599 & 11.70 & $\mathrm{OC}$ & 22 & 0.32 & 1.11 & $\ldots$ & $\ldots$ & $\ldots$ & RG & Gau13 & & Triple (ETVs) \\
\hline 9207508 & 6718 & 13.71 & SD & 2 & 2.02 & 54.47 & 0.50 & 0.95 & 23.1 & $\delta$ Sct or Tidal & Sumin Tang PhD & $\mathrm{Y}$ & \\
\hline 9236858 & 6510 & 13.04 & $\mathrm{D}$ & 2 & 2.54 & 22.82 & 0.50 & 1.12 & 14.0 & $\gamma$ Dor, $\delta \operatorname{Sct}(?)$ & Kju16b & $\mathrm{Y}$ & \\
\hline 9246715 & 4699 & 9.27 & $\mathrm{D}$ & 2 & 171.28 & 17.73 & 0.71 & 1.06 & 3.2 & RG & Gau13, Raw16 & & $\mathrm{RG}+\mathrm{RG}$ \\
\hline 9285587 & 8008 & 12.93 & ELL & 0 & 1.81 & 1.15 & $\ldots$ & $\ldots$ & $\ldots$ & $\gamma$ Dor, $\delta$ Sct & Fai15 & & $\mathrm{dA}+\mathrm{WD}, T_{\text {eff }}$ hot \\
\hline 9288175 & 6972 & 12.55 & ELL & 0 & 0.26 & 0.58 & $\ldots$ & $\ldots$ & $\ldots$ & $\gamma$ Dor, $\delta$ Sct? & & $\mathrm{Y}$ & Sparse spectrum \\
\hline 9291368 & 7889 & 14.01 & $\mathrm{SD}$ & 2 & 3.80 & 53.34 & 0.50 & 1.02 & 19.0 & $\delta$ Sct & Bra15 & $\mathrm{Y}$ & \\
\hline 9343862 & 7709 & 15.01 & SD & 2 & 1.12 & 37.32 & 0.50 & 0.87 & 21.7 & $\delta$ Sct? & Bra15 & $\mathrm{Y}$ & \\
\hline 9408183 & 4896 & 13.18 & $\mathrm{D}$ & 0 & 49.68 & 0.04 & $\ldots$ & $\ldots$ & $\ldots$ & RG & Bck14 & & $\mathrm{HB}$ \\
\hline 9426970 & 6577 & 13.23 & ELL & 0 & 1.77 & 0.99 & $\ldots$ & $\ldots$ & $\ldots$ & $\delta$ Sct & & $\mathrm{Y}$ & \\
\hline 9468382 & 4911 & 13.57 & $\mathrm{D}$ & 2 & 11.08 & 0.18 & $\ldots$ & $\ldots$ & $\cdots$ & RG & & $\mathrm{Y}$ & Likely FP (shallow ecl) \\
\hline 9470054 & 7794 & 11.72 & ELL & 0 & 1.47 & 1.78 & $\cdots$ & $\ldots$ & $\cdots$ & $\delta$ Sct & & $\mathrm{Y}$ & \\
\hline 9475663 & 7790 & 12.49 & $\mathrm{OC}$ & 2 & 0.71 & 4.14 & 0.51 & $\ldots$ & $\ldots$ & $\delta$ Sct & & $\mathrm{Y}$ & \\
\hline 9480516 & 7051 & 13.39 & ELL & 0 & 1.43 & 0.64 & $\ldots$ & $\cdots$ & $\cdots$ & $\gamma \operatorname{Dor}(?), \delta$ Sct & & $\mathrm{Y}$ & \\
\hline 9480977 & 7289 & 12.30 & ELL & 0 & 0.87 & 0.31 & $\cdots$ & $\cdots$ & $\cdots$ & $\gamma$ Dor, $\delta$ Sct & & $\mathrm{Y}$ & Sparse spectrum \\
\hline 9533489 & $\ldots$ & 12.96 & $\mathrm{D}$ & 1 & 197.15 & 0.47 & $\cdots$ & $\cdots$ & $\cdots$ & $\gamma$ Dor, $\delta$ Sct & $\operatorname{Bog} 15$ & & \\
\hline 9540226 & 4584 & 11.67 & $\mathrm{D}$ & 2 & 175.46 & 2.31 & 0.26 & 0.99 & 5.7 & RG & Gau13, Bck14, Bro18, The18 & & SB2 \\
\hline 9592855 & 7290 & 12.22 & $\mathrm{SD}$ & 2 & 1.22 & 16.09 & 0.50 & 0.98 & 20.5 & $\gamma$ Dor, $\delta$ Sct & Guo17b & & \\
\hline 9602542 & 8037 & 13.89 & ELL & 2 & 1.46 & 1.71 & 0.50 & $\ldots$ & $\ldots$ & $\gamma$ Dor, $\delta$ Sct & & $\mathrm{Y}$ & $T_{\text {eff hot }}$ \\
\hline 9612468 & 7202 & 11.53 & ELL & 0 & 0.13 & 0.10 & $\ldots$ & $\cdots$ & $\cdots$ & $\gamma$ Dor, $\delta$ Sct & Dem15 & $\mathrm{Y}$ & $\begin{array}{l}\text { Very short } P_{\text {orb }}: \text { FP, triple? } \\
\text { Circumbin. pla. }\end{array}$ \\
\hline 9637265 & 7424 & 13.93 & ELL & 0 & 1.86 & 2.75 & $\ldots$ & $\ldots$ & $\ldots$ & $\delta$ Sct & & $\mathrm{Y}$ & \\
\hline 9651298 & 7677 & 13.46 & ELL & 0 & 2.16 & 3.56 & $\ldots$ & $\ldots$ & $\cdots$ & $\delta$ Sct & & $\mathrm{Y}$ & \\
\hline 9724080 & 7470 & 13.90 & ELL & 0 & 1.17 & 5.91 & $\cdots$ & $\cdots$ & $\cdots$ & $\gamma$ Dor, $\delta$ Sct & Con 14 & $\mathrm{Y}$ & Triple (ETVs) \\
\hline 9777062 & 7466 & 12.24 & $\mathrm{D}$ & 2 & 19.23 & 25.89 & 0.60 & 1.38 & 2.8 & $\gamma$ Dor & Snd16 & & $\mathrm{Am}+\gamma$ Dor \\
\hline 9786821 & $\ldots$ & 11.51 & $\mathrm{D}$ & 1 & 21.1221 .12 & 0.03 & $\ldots$ & $\ldots$ & $\ldots$ & Tidal? & & $\mathrm{Y}$ & $\mathrm{HB}$, spots \\
\hline 9788457 & 7939 & 13.01 & SD & 2 & 0.96 & 58.46 & 0.50 & 0.77 & 26.6 & $\delta$ Sct & Brk16 & $\mathrm{Y}$ & Triple (ETVs) \\
\hline 9843435 & 7291 & 14.78 & SD & 2 & 1.68 & 37.13 & 0.50 & 1.09 & 30.0 & $\delta$ Sct & Bra15 & $\mathrm{Y}$ & \\
\hline 9850387 & 6808 & 13.55 & $\mathrm{D}$ & 2 & 2.75 & 17.49 & 0.50 & 1.01 & 13.4 & $\gamma$ Dor, $\delta$ Sct, Rossby(?) & & $\mathrm{Y}$ & \\
\hline 9851944 & 6204 & 11.25 & SD & 2 & 2.16 & 20.75 & 0.50 & 1.00 & 23.0 & $\gamma$ Dor, $\delta$ Sct & Guo16 & & $T_{\text {eff }}$ cool for $\delta$ Sct \\
\hline
\end{tabular}


Table A.1. continued.

\begin{tabular}{|c|c|c|c|c|c|c|c|c|c|c|c|c|c|}
\hline KIC & $\begin{array}{l}T_{\text {eff }} \\
{[\mathrm{K}]} \\
\end{array}$ & $K_{\text {mag }}$ & Type & $\mathrm{Ecl}$ & $\begin{array}{l}P_{\text {orb }} \\
\text { [day] }\end{array}$ & $\begin{array}{l}\text { Depth } \\
{[\%]}\end{array}$ & Sep & $\frac{W_{2}}{W_{1}}$ & $\begin{array}{c}\frac{W_{1}+W_{2}}{P_{\text {orb }}} \\
{[\%]} \\
\end{array}$ & Puls type & Literature & New PB & Notes \\
\hline 9898401 & 7376 & 12.07 & ELL & 0 & 0.15 & 0.28 & $\ldots$ & $\ldots$ & $\ldots$ & $\gamma$ Dor, $\delta$ Sct, Tidal? & & $\mathrm{Y}$ & Very short $P_{\text {orb }}:$ FP, triple? \\
\hline 9904059 & 4940 & 13.61 & $\mathrm{D}$ & 1 & 102.97 & 0.15 & $\ldots$ & $\ldots$ & $\ldots$ & $\mathrm{RG}$ & Ben+ & & SB1 \\
\hline 9953894 & 7295 & 11.08 & OC & 2 & 1.38 & 13.55 & 0.50 & $\ldots$ & $\ldots$ & $\gamma$ Dor, $\delta$ Sct & & $\mathrm{Y}$ & \\
\hline 9955262 & 6478 & 10.14 & $\mathrm{D}$ & 1 & 77.48 & 0.14 & $\ldots$ & $\ldots$ & $\ldots$ & $\gamma$ Dor? & Bor11 & $\mathrm{Y}$ & Noisy. Exoplanet? \\
\hline 9970396 & 4716 & 11.45 & $\mathrm{D}$ & 2 & 235.30 & 6.21 & 0.41 & 1.15 & 2.9 & RG & Gau13, Gau16 & & SB2 \\
\hline 10001167 & 4683 & 10.05 & $\mathrm{D}$ & 2 & 120.39 & 2.24 & 0.59 & 1.07 & 7.7 & RG & Gau13, Gau16 & & SB2 \\
\hline 10015516 & 5157 & 10.70 & $\mathrm{D}$ & 2 & 67.69 & 10.83 & 0.50 & 0.91 & 6.5 & $\mathrm{RG}+\gamma$ Dor & Ben+ & & SB1 \\
\hline 10031808 & $\ldots$ & 9.56 & $\mathrm{D}$ & 2 & 8.59 & 25.82 & 0.49 & 1.54 & 9.4 & $\gamma$ Dor? & Hel17b & & Triple (SB3) \\
\hline 10074700 & 5066 & 14.62 & $\mathrm{D}$ & 2 & 365.65 & 3.07 & 0.49 & 1.37 & 0.9 & RG & Ben+ & & SB1 \\
\hline 10092506 & 6526 & 11.21 & $\mathrm{D}$ & 0 & 31.04 & 0.05 & $\ldots$ & $\ldots$ & $\ldots$ & $\delta$ Sct, Tidal & $\operatorname{Dim} 17$ & & $\mathrm{HB}, P_{\text {orb }}$ harm., SB1 \\
\hline 10149845 & $\ldots$ & 12.10 & ELL & 0 & 4.06 & 4.51 & $\ldots$ & $\ldots$ & $\ldots$ & $\gamma$ Dor, $\delta$ Sct & & $\mathrm{Y}$ & \\
\hline 10275747 & 7362 & 12.80 & SD & 2 & 0.66 & 53.88 & 0.50 & 0.78 & 27.8 & $\gamma$ Dor, $\delta$ Sct & & $\mathrm{Y}$ & \\
\hline 10275887 & $\ldots$ & 13.04 & $\mathrm{D}$ & 2 & 9.73 & 43.23 & 0.50 & 0.94 & 12.9 & $\delta$ Sct & & $\mathrm{Y}$ & \\
\hline 10383620 & 7470 & 12.83 & SD & 2 & 0.73 & 23.27 & 0.50 & 1.01 & 33.2 & $\delta$ Sct & Brk16 & Y & Triple (ETVs) \\
\hline 10417135 & 8421 & 13.25 & ELL & 0 & 1.20 & 2.49 & $\ldots$ & $\ldots$ & $\ldots$ & $\gamma$ Dor, $\delta$ Sct & Zho10 & & $T_{\text {eff }}$ hot \\
\hline 10417986 & $\ldots$ & 9.13 & ELL & 0 & 0.07 & 0.04 & $\ldots$ & $\ldots$ & $\ldots$ & $\gamma$ Dor, $\delta$ Sct & & $\mathrm{Y}$ & Very short $P_{\text {orb }}:$ FP, triple? \\
\hline 10454725 & 6911 & 14.24 & SD & 2 & 0.83 & 32.61 & 0.50 & 1.04 & 41.4 & $\gamma$ Dor, $\delta \mathrm{Sct}$ & & $\mathrm{Y}$ & Low-amplitude spectrum \\
\hline 10485250 & 4957 & 15.79 & $\mathrm{D}$ & 2 & 16.47 & 0.72 & $\ldots$ & $\ldots$ & $\cdots$ & RG & & Y & $\begin{array}{l}T_{2}<T_{1} \text {, spots, low SNR osc., } \\
\text { sec. ecl. }\end{array}$ \\
\hline 10486425 & 7018 & 12.46 & $\mathrm{D}$ & 2 & 5.27 & 10.39 & 0.50 & 1.02 & 6.5 & $\gamma$ Dor & AS14 & & \\
\hline 10491544 & 4835 & 13.44 & $\mathrm{D}$ & 2 & 22.77 & 3.08 & 0.71 & 1.09 & 3.1 & $\mathrm{RG}$ & Cou11 & $\mathrm{Y}$ & $T_{2} \sim T_{1}$ \\
\hline 10556068 & 7901 & 11.56 & ELL & 0 & 2.12 & 1.71 & $\ldots$ & $\ldots$ & $\ldots$ & $\gamma$ Dor, $\delta$ Sct & & $\mathrm{Y}$ & Sparse, low SNR \\
\hline 10581918 & 7252 & 12.80 & $\mathrm{SD}$ & 2 & 1.80 & 72.62 & 0.50 & 0.92 & 18.9 & $\delta$ Sct & Lia17 & & \\
\hline 10614012 & 4859 & 9.71 & $\mathrm{D}$ & 1 & 132.17 & 1.00 & $\ldots$ & $\ldots$ & $\ldots$ & $\mathrm{RG}$ & Bck14 & & HB \\
\hline 10619109 & 7028 & 11.70 & SD & 2 & 2.05 & 26.88 & 0.50 & 1.05 & 21.2 & $\delta$ Sct? & Lia17 & & \\
\hline 10661783 & 7887 & 9.59 & SD & 2 & 1.23 & 21.78 & 0.50 & 1.08 & 33.0 & $\gamma$ Dor, $\delta$ Sct & Sou11, Leh13 & & \\
\hline 10684673 & 7106 & 11.12 & ELL & 0 & 0.19 & 1.06 & $\ldots$ & $\ldots$ & $\ldots$ & $\gamma$ Dor, $\delta$ Sct & TM16, Tur19 & & $\begin{array}{l}\text { Sparse spectrum, Very short } \\
P_{\text {orb }}: \text { FP, triple? }\end{array}$ \\
\hline 10735331 & 6694 & 13.38 & $\mathrm{D}$ & 1 & 213.40 & 1.29 & $\ldots$ & $\ldots$ & $\ldots$ & $\gamma$ Dor, Rossby(?) & San16 & $\mathrm{Y}$ & \\
\hline 10735519 & 4881 & 11.78 & $\mathrm{SD}$ & 1 & 0.91 & 0.19 & $\ldots$ & $\ldots$ & $\ldots$ & RG & Zho10, Gau13, Bel19 & & Likely FP (shallow ecl) \\
\hline 10736223 & 7797 & 13.65 & SD & 2 & 1.11 & 69.23 & 0.50 & 0.93 & 21.6 & $\delta$ Sct & Con14, Gie15 & $\mathrm{Y}$ & Triple (ETVs) \\
\hline 10789421 & $\ldots$ & 11.85 & ELL & 0 & 0.78 & 0.54 & $\ldots$ & $\ldots$ & $\ldots$ & $\gamma$ Dor & & $\mathrm{Y}$ & \\
\hline 10809677 & 4995 & 13.94 & $\mathrm{D}$ & 2 & 7.04 & 0.53 & 0.50 & 0.94 & 3.6 & RG & Zho10, Cou11, Gau13 & & Likely FP (shallow ecl) \\
\hline 10858117 & 5354 & 14.32 & $\mathrm{D}$ & 2 & 606.11 & 16.51 & 0.08 & 1.36 & 0.3 & RG? & & $\mathrm{Y}$ & Low SNR, high ecc. \\
\hline 10905804 & 8000 & 14.42 & $\mathrm{SD}$ & 1 & 0.75 & 17.00 & $\ldots$ & $\ldots$ & $\ldots$ & $\delta$ Sct & Con 14 & $\mathrm{Y}$ & Triple (ETVs) \\
\hline 10920813 & $\ldots$ & 13.76 & $\mathrm{D}$ & 2 & 53.74 & 31.75 & 0.46 & 1.25 & 5.6 & RG & & $\mathrm{Y}$ & $T_{2}>T_{1}, \ell=1$ depleted \\
\hline 10972830 & $\ldots$ & 10.17 & ELL & 0 & 0.68 & 0.02 & $\ldots$ & $\ldots$ & $\ldots$ & Tidal? & & $\mathrm{Y}$ & True binary? (very shallow ELL) \\
\hline 10979669 & 6697 & 12.33 & ELL & 0 & 0.93 & 6.47 & $\ldots$ & $\ldots$ & $\ldots$ & $\gamma$ Dor, $\delta$ Sct? & & $\mathrm{Y}$ & \\
\hline 10989032 & 8620 & 13.87 & $\mathrm{SD}$ & 2 & 2.31 & 0.95 & 0.50 & 1.36 & 5.9 & $\delta$ Sct & ZXB17 & & $\mathrm{WD}+\delta \mathrm{Sct}$ \\
\hline 10991989 & 5021 & 10.28 & SD & 2 & 0.97 & 0.88 & 0.50 & 0.93 & 18.3 & RG & Gau13, Hel16 & & Triple (ETVs) \\
\hline 11044668 & 4959 & 12.35 & $\mathrm{D}$ & 0 & 139.45 & 0.31 & $\ldots$ & $\ldots$ & $\ldots$ & RG & Bck14 & & $\mathrm{HB}$ \\
\hline 11135978 & 5004 & 12.33 & $\mathrm{OC}$ & 22 & 0.29 & 0.76 & $\ldots$ & $\ldots$ & $\ldots$ & RG & Zho10, Gau13 & & Triple (ETVs)? \\
\hline 11147460 & 4855 & 13.91 & $\mathrm{D}$ & 2 & 4.11 & 0.47 & 0.50 & 1.02 & 8.6 & RG & Zho10, Gau13, Lur17 & & Likely FP, asynchronous EB \\
\hline 11179657 & $\ldots$ & 17.07 & ELL & 0 & 0.79 & 1.80 & $\ldots$ & $\ldots$ & $\ldots$ & sdB g-modes & Pab12 & & \\
\hline 11180361 & 8330 & 7.75 & SD & 1 & 0.53 & 0.16 & $\ldots$ & $\ldots$ & $\ldots$ & $\gamma$ Dor, $\delta$ Sct & Mur18 & & $T_{\text {eff }}$ hot \\
\hline 11197853 & 4981 & 13.59 & $\mathrm{D}$ & 1 & 0.70 & 0.01 & $\ldots$ & $\ldots$ & $\ldots$ & RG & Gau13 & & FP (very shallow ecl.) \\
\hline 11285625 & 6882 & 10.14 & $\mathrm{D}$ & 2 & 10.79 & 11.57 & 0.50 & 0.98 & 5.0 & $\gamma$ Dor & Deb13 & & \\
\hline 11295347 & 7620 & 11.73 & ELL & 0 & 0.89 & 1.87 & $\ldots$ & $\ldots$ & $\ldots$ & $\gamma$ Dor, $\delta$ Sct & & $\mathrm{Y}$ & \\
\hline 11401845 & 7590 & 14.36 & $\mathrm{SD}$ & 2 & 2.16 & 41.04 & 0.50 & 1.00 & 20.3 & $\delta$ Sct, Tidal & Lee 17 & & \\
\hline 11408810 & 7555 & 12.61 & $\mathrm{OC}$ & 0 & 0.75 & 1.08 & $\ldots$ & $\ldots$ & $\ldots$ & $\gamma$ Dor, Rossby(?) & & $\mathrm{Y}$ & \\
\hline 11494130 & 6330 & 10.99 & $\mathrm{D}$ & 0 & 18.96 & 0.06 & $\ldots$ & $\ldots$ & $\ldots$ & Tidal & Tho12, Smu15 & & $\mathrm{HB}, P_{\text {orb }}$ harm., SB1 \\
\hline 11566064 & 6679 & 13.37 & $\mathrm{D}$ & 1 & 152.11 & 0.38 & $\ldots$ & $\ldots$ & $\ldots$ & $\gamma$ Dor? & DR12 & $\mathrm{Y}$ & Low SNR, planet candidate \\
\hline 11572363 & 6069 & 12.43 & $\mathrm{D}$ & 0 & 19.03 & $\ldots$ & $\ldots$ & $\ldots$ & $\ldots$ & Tidal & & $\mathrm{Y}$ & $\mathrm{HB}, P_{\text {orb }}$ harm. \\
\hline 11671429 & 7363 & 10.97 & $\mathrm{D}$ & 2 & 112.46 & 23.26 & 0.73 & 0.62 & 1.7 & $\gamma$ Dor, $\delta$ Sct & Uyt11 & & \\
\hline 11768970 & 5038 & 12.66 & $\mathrm{D}$ & 2 & 15.54 & 0.95 & 0.65 & 2.23 & 1.9 & RG & Cou11 & $\mathrm{Y}$ & Grazing eclipses \\
\hline 11817750 & 6930 & 12.25 & $\mathrm{D}$ & 2 & 9.76 & 3.85 & 0.50 & 1.08 & 3.9 & $\gamma$ Dor & Lur17 & & \\
\hline 11820830 & 7007 & 12.09 & $\mathrm{D}$ & 1 & 12.73 & 1.14 & $\ldots$ & $\ldots$ & $\ldots$ & $\gamma$ Dor & & Y & HB \\
\hline 11825198 & $\ldots$ & $\ldots$ & $\mathrm{OC}$ & 0 & 2.16 & 1.56 & $\ldots$ & $\ldots$ & $\ldots$ & $\gamma$ Dor, $\delta$ Sct, Rossby(?) & & $\mathrm{Y}$ & \\
\hline 11874338 & 5041 & 13.93 & $\mathrm{D}$ & 2 & 15.98 & 0.06 & $\ldots$ & $\ldots$ & $\ldots$ & RG & Gau13 & & FP (very shallow ecl.) \\
\hline 11923819 & 7724 & 11.56 & $\mathrm{D}$ & 2 & 33.16 & 21.09 & 0.34 & 1.31 & 2.2 & $\gamma$ Dor & Lur17 & & \\
\hline 11968514 & 4940 & 11.45 & $\mathrm{D}$ & 2 & 1.04 & 0.00 & 0.50 & 1.00 & 17.9 & RG & Zho10, Gau13 & & Likely FP (shallow ecl) \\
\hline 11973705 & 7404 & 9.12 & ELL & 0 & 6.77 & 0.75 & $\ldots$ & $\ldots$ & $\ldots$ & $\gamma$ Dor, $\delta$ Sct, Rossby(?) & Bal11 & & $\mathrm{SPB}+\delta \mathrm{Sct}$ \\
\hline 12071006 & 7338 & 13.53 & $\mathrm{SD}$ & 2 & 6.10 & 88.68 & 0.50 & 1.00 & 19.1 & $\gamma$ Dor, $\delta$ Sct & Con14, Gie15 & $\mathrm{Y}$ & Triple (ETVs) \\
\hline 12167361 & 8017 & 10.38 & $\mathrm{D}$ & 1 & 47.93 & 0.21 & $\ldots$ & $\ldots$ & $\ldots$ & $\gamma$ Dor & Lur17 & & $T_{\text {eff }}$ hot, KOI 980 \\
\hline 12216706 & 8530 & 15.01 & SD & 2 & 1.47 & 16.48 & 0.50 & 0.75 & 15.1 & $\delta$ Sct & & $\mathrm{Y}$ & $T_{\text {eff }}$ hot \\
\hline 12216817 & 6681 & 10.66 & ELL & 0 & 0.25 & 4.10 & $\ldots$ & $\ldots$ & $\ldots$ & $\gamma$ Dor, $\delta$ Sct & TM16 & & \\
\hline 12268220 & 7826 & 11.43 & $\mathrm{D}$ & 2 & 4.42 & 4.47 & $\ldots$ & $\ldots$ & $\ldots$ & $\gamma$ Dor, $\delta$ Sct & & $\mathrm{Y}$ & \\
\hline 12367310 & 4965 & 13.84 & $\mathrm{D}$ & 2 & 8.63 & 3.44 & 0.51 & 1.12 & 4.1 & RG & & $\mathrm{Y}$ & Triple (ETVs), spots \\
\hline 12470041 & 7290 & 13.41 & $\mathrm{D}$ & 1 & 14.67 & 2.90 & $\ldots$ & $\ldots$ & $\ldots$ & $\gamma$ Dor, Tidal(?) & & $\mathrm{Y}$ & $\mathrm{HB}$ \\
\hline 12645761 & 4844 & 13.37 & $\mathrm{D}$ & 2 & 5.425 .42 & 1.25 & 0.50 & 1.10 & 8.2 & $\mathrm{RG}$ & Zho10, Gau13 & & FP or triple \\
\hline 12785282 & 6924 & 13.52 & $\mathrm{SD}$ & 2 & 0.79 & 15.40 & 0.50 & 0.96 & 26.0 & $\gamma$ Dor & & $\mathrm{Y}$ & \\
\hline
\end{tabular}

